\title{
Evaluating the Effect of Self-Interference on the Performance of Full-Duplex Two-Way Relaying Communication with Energy Harvesting
}

\author{
Phong Nguyen-Huu ${ }^{1}$, Khuong Ho-Van ${ }^{1}$, Vo Nguyen Quoc Bao ${ }^{2}$ \\ ${ }^{1}$ Ho Chi Minh City University of Technology, VNU-HCM, Ho Chi Minh City, Vietnam \\ 2 Posts and Telecommunications Institute of Technology, Ho Chi Minh City, Vietnam
}

Correspondence: Khuong Ho-Van, hvkhuong@hcmut.edu.vn

Communication: received 14 June 2019, revised 13 August 2019, accepted 15 August 2019

Online publication: 23 November 2019, Digital Object Identifier: 10.21553/rev-jec.240

The associate editor coordinating the review of this article and recommending it for publication was Prof. Nguyen Le Hung.

\begin{abstract}
In this paper, we study the throughput and outage probability (OP) of two-way relaying (TWR) communication system with energy harvesting (EH). The system model consists two source nodes and a relay node which operates in full-duplex (FD) mode. The effect of self-interference (SI) due to the FD operation on the system performance is evaluated for both one-way full duplex (OWFD) and two-way full duplex (TWFD) diagrams where the amplify-and-forward (AF) relay node collects energy harvesting with the time switching (TS) scheme. We first propose an individual OP expression for each specific source. Then, we derive the exact closed-form overall OP expression for the OWFD diagram. For the TWFD diagram, we propose an approximate closed-form expression for the overall OP. The overall OP comparison among hybrid systems (Two-Way Half-Duplex (TWHD), OWFD, TWFD) are also discussed. Finally, the numerical/simulated results are presented for Rayleigh fading channels to demonstrate the correction of the proposed analysis.
\end{abstract}

Keywords- Two-way relaying communications, Relaying, Full-duplex, Energy harvesting.

\section{INTRODUCTION}

The spectral efficiency is an important system specification for designing next-generation wireless networks. To address spectral efficiency problem, some works proposed the cognitive radio technique in the twoway $^{1}$ relaying network [1-4]. However, almost current wireless systems are operating in half-duplex (HD) mode with different frequencies for down-link and uplink channels. Recently, full-duplex (FD) transmission had been proposed with the promise of significant improvements in spectral efficiency due to shared same frequency and time slot $[5,6]$. However, SI caused by simultaneous transceiver operation of the FD mode affects the system performance [7]. To evaluate the effect of SI on the OWFD and TWFD systems, the authors [8] proposed the analysis on the average endto-end rate and the OP. Compared to the OWFD, the TWFD achieves higher spectrum efficiency but suffers more SI [9]. Moreover, EH from radio frequency signals is an emerging technology helping prolong the lifetime of wireless devices. EH was proposed for internet of things (IoT) applications [10] and 5G full-duplex communications [11-13]. As such, FD communication system with EH can obtain both high spectral efficiency and high energy efficiency.

\footnotetext{
${ }^{1}$ All the works in $[1-4]$ did not mention the full-duplex relaying.
}

\subsection{Related Works}

This section conducts the survey of the (OWFD, TWFD) communications systems with EH. The OWFD communications in cooperative relaying networks with $\mathrm{EH}$ was considered in the recent works. The authors in [14] studied the influence of SI on the OWFD transmission where the optimal protocol was proposed to choose either the TWHD or the OWFD with the AF relay to minimize the OP. The selected AF relay to maximize the information rate subject to the total power limitation was proposed in [15] where the optimal transmit power can be obtained by Lagrangian multiplier method. Considering the AF and decodeand-forward (DF) operations, the authors [16] analyzed the OP combined with the selection of relay nodes to compare with direct links under the imperfect channel state information (CSI). Analyzing the individual OPs with the relay node using $\mathrm{AF}$ and $\mathrm{DF}$ techniques for comparison between FD and HD was performed in [17] but only simulations were demonstrated for the $\alpha-\mu$ fading model. Optimizing the OP and quality of services (QoS) for non-linear EH models was implemented in [18] where the proposed FD DF relaying model was deduced by the optimal solution based on the golden section method. The authors in [19] solves the power efficiency optimization problem for EH FD relaying with the joint power and time allocation scheme to obtain different source transmit powers. While [20] proposed 
the optimum transmission algorithm with significantly reduced complexity, [21] optimized channel capacity. In [22], the beam-former design to maximize the signalto-noise ratio (SNR) for a DF relay was implemented but only simulations were shown to prove that the multi-antenna relay performs better than the singleantenna relay. An optimization algorithm proposed for the multiple-input multiple-output (MIMO) orthogonal frequency division multiplexing (OFDM) networks to achieve spectral efficiency for OWFD networks was conducted in [23]. Finally, the analysis on the OP and the throughput in the FD cognitive radio networks was carried out in [24].

In TWFD systems, the SI caused by the FD operation was available at all nodes. The authors in [25] analyzed the exact individual OP for each node for the AF relay. The design of energy signal and decoder for TWFD networks was studied in [26] and the sum-throughput comparison between the TWFD and the TWHD was also presented there. The authors in [27] proposed two schemes called relay selection (RS) and all-participant (AP) to optimize the power splitting factor in order to minimize the OP and maximize the sum capacity. The simulation results in [27] illustrated the sum capacity of the RS higher than that of the AP. Bit error rate (BER) analysis with spatial diversity was studied in [28] and it is noticeable that when the quality of the SI cancellation is improved, the BER performance of FD is better than HD. Then, the analysis of the individual OP with the DF relay was carried out in [29] and [30] where the imperfect CSI was also considered. The authors in [31] proposed the optimal power allocation scheme and the optimal relay node placement strategy to minimize the OP for the AF relay but did not perform the closedform analysis. The beam-forming design to optimize the time division ratio for EH FD networks was studied in [32]. To assess the effect of SI on TWFD systems, the authors in [33] proposed a two-node model to exchange information through multiple relay nodes, using AF technique, TS and power splitting (PS) methods. The analysis of individual OP and specific throughput for each node was also studied there. To implement the overall OP, the authors further proposed the analysis at approximately high SNRs for Rayleigh fading channels.

\subsection{Motivation and Contribution}

The above survey exposes that the effect of SI on the performance of the TWFD communication system with energy harvesting has not been fully evaluated yet, especially for the overall OP. Also, the spectral efficiency needs to be compared and evaluated among different diagrams (TWFD, OWFD, TWHD).

The contributions of this paper can be summarized as follows:

1) Propose the overall exact closed-form OP expression for the OWFD communication system.

2) Suggest the overall approximate closed-form OP expression for the TWFD communication system.

3) Compare and evaluate the effect of SI on the system performance in terms of OP and throughput for three diagrams: TWFD, OWFD, and TWHD.

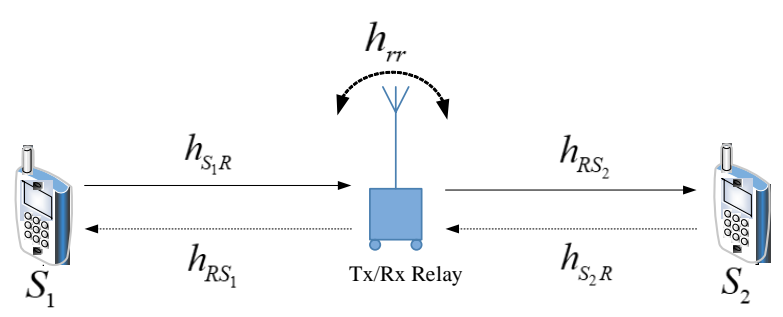

(a) One-Way Full-Duplex relaying

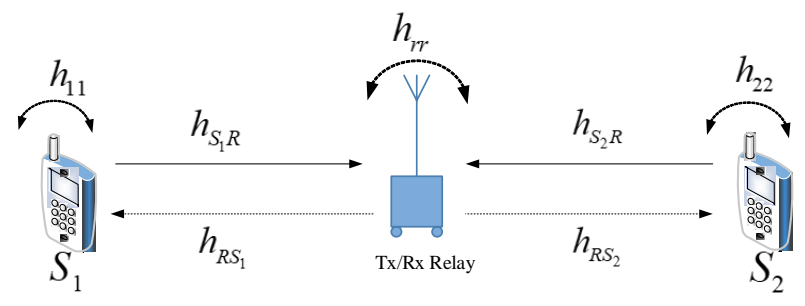

(b) Two-Way Full-Duplex relaying

Figure 1. OWFD and TWFD system models.

The rest of the paper is organized as follows. In Section 2, we describe the system model. Section 3 presents a detailed performance analysis. The results are presented in Section 4 whilst the conclusions are given in Section 5.

\section{System Model}

Figure 1(a) shows the OWFD system model. The relay $R$ has the limited power; therefore, $R$ collects radio frequency (RF) energy from $S_{1}$ or $S_{2}$ in the first time slot of $\alpha T$. For the TWFD communications in Figure 1(b), $R$ collects energy from both $S_{1}$ and $S_{2}$. It is noted that for the OWFD communications, only $R$ operates in the FD mode while for the TWFD communications, all three nodes operate the FD mode.

We define the residual SI channels at $S_{1}$ as $h_{11}$, at $S_{2}$ as $h_{22}$, and at $R$ as $h_{r r}$. The corresponding SI can be modeled as a Gaussian random variable with zero mean and variance of $\sigma_{11}^{2}=\sigma_{22}^{2}=\sigma_{r r}^{2}=\sigma_{S I}^{2}$ as in $[6,9,33]$.

The involved channels, $S_{1} \rightarrow R$ and $S_{2} \rightarrow R$, are denoted as $h_{S_{1} R}$ and $h_{S_{2} R}$, respectively. The coefficients for $R \rightarrow S_{1}$ and $R \rightarrow S_{2}$ channels are also signified as $h_{R S_{1}}$ and $h_{R S_{2}}$, correspondingly. We assume that channel coefficients are independent and the incoming and outgoing channels are reciprocal, i.e., $h_{S_{1} R}=h_{R S_{1}}=h_{1}$, $h_{S_{2} R}=h_{R S_{2}}=h_{2}$, with the block Rayleigh fading distribution. Therefore, $X=\left|h_{1}\right|^{2}$ and $Y=\left|h_{2}\right|^{2}$ are the random variables (RVs), with exponential distributions, i.e., they have the probability distribution functions (PDFs), $f_{X}(x)=\lambda_{1} e^{-\lambda_{1} x}, f_{Y}(y)=\lambda_{2} e^{-\lambda_{2} y}$ and the cumulative distribution functions (CDFs), $F_{X}(x)=1-$ $e^{-\lambda_{1} x}, F_{Y}(y)=1-e^{-\lambda_{2} y}$. Here, the expectation of $X$ or $Y$ is denoted as $\mu_{i}=\frac{1}{\lambda_{i}}=d_{i}^{-\chi}$ with $\chi$ being the path-loss exponent and $d_{i}$ being the transmitter-receiver distance, $i=1,2$. 


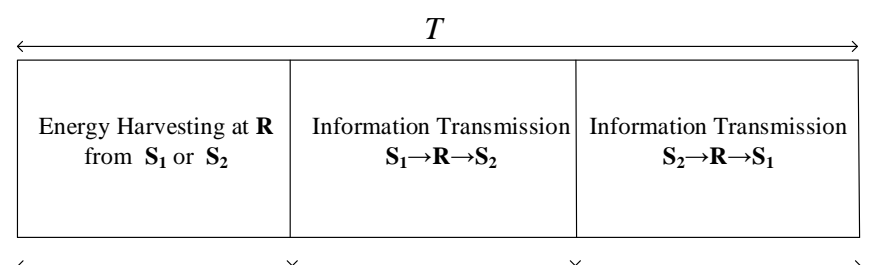

$\alpha T$

$(1-\alpha) T / 2$

$(1-\alpha) T / 2$

(a) One-Way FD Relaying

\begin{tabular}{|c|c|}
\hline $\begin{array}{c}\text { Energy Harvesting at } \mathbf{R} \\
\text { from } \mathbf{S}_{\mathbf{1}} \& \mathbf{S}_{\mathbf{2}}\end{array}$ & $\begin{array}{c}\text { Information Transmission } \\
\mathbf{S}_{\mathbf{1}}, \mathbf{S}_{\mathbf{2}} \rightarrow \mathbf{R} \text { and } \mathbf{R} \rightarrow \mathbf{S}_{\mathbf{1}}, \mathbf{S}_{\mathbf{2}}\end{array}$ \\
\hline$\alpha T$ & $\times$
\end{tabular}

(b) Two-way FD Relaying

Figure 2. Time Switching Protocol at AF Full-Duplex Relaying.

\subsection{SNR in the OWFD Communications}

From Figure 2(a), the energy collected in the first time-slot of $\alpha T$ is

$$
E_{R}=\beta\left(P_{1}\left|h_{1}\right|^{2}+\sigma_{R}^{2}\right) \alpha T .
$$

Similarly, if $R$ only collects the energy from $S_{2}$, its collected energy is $E_{R}=\beta\left(P_{2}\left|h_{2}\right|^{2}+\sigma_{R}^{2}\right) \alpha T$. Here, $P_{1}$ and $P_{2}$ are transmit powers of $S_{1}$ and $S_{2}$, respectively; $0<\beta<1$ is the energy conversion coefficient; $0<\alpha<$ 1 is the time switching ratio; $T$ is the block time.

From (1), the transmit power at the $R$ is

$$
\begin{aligned}
P_{R} & =\frac{E_{R}}{(1-\alpha) T} \\
& =\frac{\alpha \beta}{(1-\alpha)}\left(P_{1}\left|h_{1}\right|^{2}+\sigma_{R}^{2}\right) \\
& =\phi\left(P_{1}\left|h_{1}\right|^{2}+\sigma_{R}^{2}\right),
\end{aligned}
$$

where $\phi=\frac{\alpha \beta}{1-\alpha}$.

$S_{1}$ and $S_{2}$ exchange information via the AF relay. In the second time-slot of $\frac{(1-\alpha) T}{2}$, the information is transmitted from $S_{1} \rightarrow R \rightarrow S_{2}$. The signal received at $R$ in the time-slot $t$ is described as

$$
y_{R}[t]=\sqrt{P_{1}} h_{1} x_{1}[t]+h_{r r} x_{R}[t]+n_{R}[t],
$$

where $E\left\{\left|x_{1}(t)\right|^{2}\right\}=1$ with $E\{\}$ being the expectation operator; $x_{1}(t)$ and $x_{R}(t)$ are the transmit signals from $S_{1}$ and $R$, respectively; $n_{R}(t)$ denotes the additive white Gaussian noise (AWGN) at $R$ with zero mean and variance $\sigma_{R}^{2}$.

For the AF based OWFD communications, the transmit signal of the relay can be expressed as in [34] and [35]

$$
x_{R}[t]=\sqrt{P_{R}} \theta_{1} y_{R}[t-1],
$$

where $\theta_{1}$ is the power constraint factor at $R$ :

$$
\theta_{1}=\frac{1}{\sqrt{P_{1}\left|h_{1}\right|^{2}+P_{R}\left|h_{r r}\right|^{2}+\sigma_{R}^{2}}} .
$$

The received signal at $S_{2}$ is

$$
y_{2}[t]=h_{2} x_{R}[t]+n_{2}(t)
$$

with $E\left\{\left|x_{R}(t)\right|^{2}\right\}=P_{R}$.

Replacing (4) and (5) into (6), we have

$$
\begin{aligned}
y_{2}[t] & =\theta_{1} \sqrt{P_{R}} h_{2} \times \\
& \left(\sqrt{P_{1}} h_{1} x_{1}[t-1]+h_{r r} x_{R}[t-1]+n_{R}[t-1]\right)+n_{2}[t] \\
& =\underbrace{\theta_{1} \sqrt{P_{R}} \sqrt{P_{1}} h_{1} h_{2} x_{1}[t-1]}_{\text {signal }}+\underbrace{\theta_{1} \sqrt{P_{R}} h_{2} h_{r r} x_{R}[t-1]}_{\text {SI }} \\
& +\underbrace{\theta_{1} \sqrt{P_{R}} h_{2} n_{R}[t-1]+n_{2}[t]}_{\text {noise }},
\end{aligned}
$$

where $n_{i}(t), i \in(1, R, 2)$, is the AWGN at $S_{1}, R, S_{2}$ with zero mean and variance $\sigma_{i}^{2}$. Without loss of generality, we let $\sigma_{1}^{2}=\sigma_{2}^{2}=\sigma_{R}^{2}=\sigma^{2}$.

From (7), the SNR at $S_{1}$ is

$$
\begin{aligned}
\gamma_{2}^{\text {OWFD }} & =\frac{E\left\{\mid \text { signal }\left.\right|^{2}\right\}}{E\left\{\mid \text { noise }\left.\right|^{2}\right\}} \\
& =\frac{\theta_{1}^{2} P_{R} P_{1}\left|h_{1}\right|^{2}\left|h_{2}\right|^{2}}{\theta_{1}^{2}\left(P_{R}\right)^{2}\left|h_{2}\right|^{2} \sigma_{r r}^{2}+\theta_{1}{ }^{2} P_{R}\left|h_{2}\right|^{2} \sigma^{2}+\sigma^{2}} .
\end{aligned}
$$

Replacing $P_{R}$ in (2) and $\theta_{1}$ in (5) into (8), we have $\gamma_{2}^{O W F D}$

$$
\begin{aligned}
& =\frac{P_{1}\left|h_{1}\right|^{2}\left|h_{2}\right|^{2}}{\left|h_{2}\right|^{2}\left(\sigma^{2}+\phi \sigma_{S I}^{2} \sigma^{2}\right)+\phi P_{1}\left|h_{1}\right|^{2}\left|h_{2}\right|^{2} \sigma_{S I}^{2}+\sigma^{2}\left(\frac{1}{\phi}+\sigma_{S I}^{2}\right)} \\
& =\frac{a_{1} x y}{b y+c_{1} x y+c},
\end{aligned}
$$

where $a_{1}=P_{1}, b=\sigma^{2}+\phi \sigma_{S I}^{2} \sigma^{2}, c_{1}=\phi P_{1} \sigma_{S I}^{2}, c=$ $\sigma^{2}\left(\frac{1}{\phi}+\sigma_{S I}^{2}\right), E\left\{\left|h_{r r}\right|^{2}\right\}=\sigma_{r r}^{2}=\sigma_{S I}^{2}$.

Please see Appendix A for detailed derivation of (9).

Using the same approach as (9), the SNR at $S_{2}$ is $\gamma_{1}^{\text {OWFD }}$

$$
\begin{aligned}
& =\frac{P_{2}\left|h_{1}\right|^{2}\left|h_{2}\right|^{2}}{\left|h_{1}\right|^{2}\left(\sigma^{2}+\phi \sigma_{S I}^{2} \sigma^{2}\right)+\phi P_{2}\left|h_{1}\right|^{2}\left|h_{2}\right|^{2} \sigma_{S I}^{2}+\sigma^{2}\left(\frac{1}{\phi}+\sigma_{S I}^{2}\right)} \\
& =\frac{a_{2} x y}{b x+c_{2} x y+c^{\prime}},
\end{aligned}
$$

where $a_{2}=P_{2}, b=\sigma^{2}+\phi \sigma_{S I}^{2} \sigma^{2}, c_{2}=\phi P_{2} \sigma_{S I}^{2}, c=$ $\sigma^{2}\left(\frac{1}{\phi}+\sigma_{S I}^{2}\right)$.

Please see Appendix A for detailed derivation of (10). 


\subsection{SNR in the TWFD Communications}

From Figure 2(b), the energy collected in the first time-slot of $\alpha T$ is

$$
E_{R}=\beta\left(P_{1}\left|h_{1}\right|^{2}+P_{2}\left|h_{2}\right|^{2}+\sigma_{R}^{2}\right) \alpha T .
$$

From (11), the transmit power at $R$ is inferred as

$$
\begin{aligned}
P_{R} & =\frac{E_{R}}{(1-\alpha) T} \\
& =\frac{\alpha \beta}{(1-\alpha)}\left(P_{1}\left|h_{1}\right|^{2}+P_{2}\left|h_{2}\right|^{2}+\sigma_{R}^{2}\right) \\
& =\phi\left(P_{1}\left|h_{1}\right|^{2}+P_{2}\left|h_{2}\right|^{2}+\sigma_{R}^{2}\right) .
\end{aligned}
$$

Unlike the OWFD communications, in the TWFD diagram, $S_{1}$ and $S_{2}$ exchange information in the same timeslot of $(1-\alpha) T$; therefore, multi-access (MA) phase and broadcast phase $(\mathrm{BC})$ can perform simultaneously in one time-slot.

The received signal at $R$ in time-slot $t$ is described as

$$
y_{R}[t]=\sqrt{P_{1}} h_{1} x_{1}[t]+\sqrt{P_{2}} h_{2} x_{2}[t]+h_{r r} x_{R}[t]+n_{R}[t],
$$

where $x_{1}(t), x_{R}(t)$, and $x_{2}(t)$ are the transmit signals of $S_{1}, R$, and $S_{2}$, respectively; $n_{R}(t)$ denotes the AWGN at $R$ with zero mean and variance $\sigma_{R}^{2}$.

For the AF based TWFD communications, in $t$-th time slot, the signal transmitted by the relay is the amplified version of the prior received signal as in $[6,9,33,36,37]$ :

$$
x_{R}[t]=\sqrt{P_{R}} \theta y_{R}[t-1] .
$$

The amplification factor at the AF relay is

$$
\theta=\frac{1}{\sqrt{P_{1}\left|h_{1}\right|^{2}+P_{2}\left|h_{2}\right|^{2}+P_{R}\left|h_{r r}\right|^{2}+\sigma_{R}^{2}}} .
$$

The received signal at $S_{1}$ is given by

$$
y_{1}[t]=h_{1} x_{R}[t]+\sqrt{P_{1}} h_{11} x_{1}[t]+n_{1}[t],
$$

where $h_{11}$ is residual SI at $S_{1}$, and $n_{1}[t]$ is the AWGN at $S_{1}$.

Replacing (14) into (16), we have

$$
\begin{aligned}
& y_{1}[t] \\
& \quad=\theta \sqrt{P_{R}}\left(\sqrt{P_{1}}\left|h_{1}\right|^{2} x_{1}[t-1]+\sqrt{P_{2}}\left|h_{1}\right|\left|h_{2}\right| x_{2}[t-1]\right) \\
& \quad+\theta \sqrt{P_{R}} h_{1} h_{r r} x_{R}[t-1]+\sqrt{P_{1}} h_{11} x_{1}[t] \\
& \quad+\theta \sqrt{P_{R}} h_{1} n_{R}[t-1]+n_{1}[t] .
\end{aligned}
$$

Suppose that the CSI is perfect. Then, in (17) the component containing $x_{2}[t-1]$ is the useful signal at $S_{1}$ while the component $x_{1}[t-1]$ is known by $S_{1}$; therefore, it can be removed. As such, (17) can be written as

$$
\begin{aligned}
y_{1}[t]= & \underbrace{\theta \sqrt{P_{R} P_{2}}\left|h_{1}\right|\left|h_{2}\right| x_{2}[t-1]}_{\text {signal }} \\
& +\underbrace{\theta \sqrt{P_{R}} h_{1} h_{r r} x_{R}[t-1]+\sqrt{P_{1}} h_{11} x_{1}[t]}_{\text {SI }} \\
& +\underbrace{\theta \sqrt{P_{R}} h_{1} h_{R}[t-1]+n_{1}[t]}_{\text {noise }},
\end{aligned}
$$

where $h_{11}$ is the SI caused by the FD operation at $S_{1}$.

From (18), one can infer the SNR at $S_{1}$ as

$\gamma_{1}^{T W F D}=\frac{\theta^{2} P_{R} P_{2}\left|h_{1}\right|^{2}\left|h_{2}\right|^{2}}{\theta^{2} P_{R}{ }^{2}\left|h_{1}\right|^{2}\left|h_{r r}\right|^{2}+\theta^{2} P_{R}\left|h_{1}\right|^{2} \sigma_{R}^{2}+P_{1}\left|h_{11}\right|^{2}+\sigma_{1}^{2}}$.

Replacing $P_{R}$ in (12) and $\theta$ in (15) into (19) and after some manipulations, we have

$$
\gamma_{1}^{\text {TWFD }}=\frac{P_{2}\left|h_{1}\right|^{2}\left|h_{2}\right|^{2}}{\left|h_{1}\right|^{2}\left\{\sigma_{S I}^{2} \phi\left(P_{1}\left|h_{1}\right|^{2}+P_{2}\left|h_{2}\right|^{2}+\sigma^{2}\right)+\sigma^{2}\right\}+k_{1}} .
$$

From (20), we have

$$
\gamma_{1}^{T W F D}=\frac{e_{1} x y}{x\left[f_{1}\left(P_{1} x+P_{2} y+g_{1}\right)+g_{1}\right]+k_{1}}
$$

where $e_{1}=P_{2}, f_{1}=\sigma_{S I}^{2} \phi, g_{1}=\sigma^{2}$, and $k_{1}=$ $\left(P_{1} \sigma_{S I}^{2}+\sigma^{2}\right)\left(\frac{1}{\phi}+\sigma_{S I}^{2}\right)$.

Please see Appendix B for detailed derivation of (21).

Following the same derivation as (21), we have

$$
\gamma_{2}^{T W F D}=\frac{e_{2} x y}{y\left[f_{2}\left(P_{1} x+P_{2} y+g_{2}\right)+g_{2}\right]+k_{2}},
$$

where $e_{2}=P_{1}, f_{2}=\sigma_{S I}^{2} \phi, g_{2}=\sigma^{2}$, and $k_{2}=$ $\left(P_{2} \sigma_{S I}^{2}+\sigma^{2}\right)\left(\frac{1}{\phi}+\sigma_{S I}^{2}\right)$.

Please see Appendix B for detailed derivation of (22).

\section{Performance Analysis}

\subsection{The OP of the OWFD Communications}

The individual OP of $S_{i}$ is defined as

$$
P_{\text {out }, i}^{\text {OWFD }}=\operatorname{Pr}\left(\gamma_{i}^{\text {OWFD }}<\tau\right)
$$

where $i \in(1,2)$, and $\tau$ is the SNR threshold at the node $S_{i}$.

Throughput can be calculated through $P_{\text {Out } i}^{O W D}$ at the fixed data rate $R_{T}$ (bps/Hz). For the OWFD communications, the throughput is given by

$$
T_{0}=R_{T}\left(1-P_{\text {out }, i}^{O W F D}\right) \frac{(1-\alpha)}{2},
$$

where $\tau=2^{R_{T}}-1$.

The OP is defined as the probability which the SNR is less than the SNR threshold:

$$
\begin{aligned}
P_{\text {out }, 1}^{\text {OWFD }} & =\operatorname{Pr}\left(\gamma_{1}^{\text {OWFD }}<\tau\right) \\
& =\operatorname{Pr}\left(\frac{a_{2} x y}{b x+c_{2} x y+c}<\tau\right) \\
& =\left\{\begin{array}{cc}
\operatorname{Pr}\left(y<\frac{\tau\{b x+c\}}{x\left(a_{2}-\tau c_{2}\right)}\right) & , a_{2}-\tau c_{2}>0 \\
1 & , a_{2}-\tau c_{2}<0
\end{array}\right.
\end{aligned}
$$

As shown in Appendix $C, P_{\text {out }, 1}^{O W F D}$ in (25) can be represented in the closed form for the case of $a_{2}-\tau c_{2}>0$ as

$$
P_{\text {out }, 1}^{\text {OWFD }}=1-\lambda_{1} e^{-\frac{\lambda_{2} \tau b}{\left(a_{2}-\tau c_{2}\right)}} \sqrt{\frac{\psi}{\lambda_{1}}} K_{1}\left(\sqrt{\psi \lambda_{1}}\right),
$$

where $\psi=\frac{4 \tau c \lambda_{2}}{\left(a_{2}-\tau c_{2}\right)}$. 
Following the same approach as (26), we have

$$
\begin{aligned}
P_{\text {out }, 2}^{\text {OWFD }} & =\operatorname{Pr}\left(\gamma_{2}^{\text {OWFD }}<\tau\right) \\
& =1-\lambda_{2} e^{-\frac{\lambda_{1} \tau b}{\left(a_{1}-\tau c_{1}\right)}} \sqrt{\frac{\vartheta}{\lambda_{2}}} K_{1}\left(\sqrt{\vartheta \lambda_{2}}\right),
\end{aligned}
$$

where $\vartheta=\frac{4 \tau c \lambda_{1}}{\left(a_{1}-\tau c_{1}\right)}$.

Please see Appendix C for detailed derivation of (27).

The end-to-end overall OP of the AF based OWFD communications is defined as

$$
\begin{aligned}
P_{e 2 e}^{\text {OWFD }} & =\operatorname{Pr}\left(\left\{\gamma_{1}^{\text {OWFD }}<\tau\right\} \cup\left\{\gamma_{2}^{\text {OWFD }}<\tau\right\}\right) \\
& =\underbrace{\operatorname{Pr}\left(\gamma_{1}^{\text {OWFD }}<\tau\right)}_{P_{\text {out }, 1}^{\text {OWFD }}}+\underbrace{\operatorname{Pr}\left(\gamma_{2}^{\text {OWFD }}<\tau\right)}_{P_{\text {out }, 2}^{\text {OWFD }}} \\
& -\underbrace{\operatorname{Pr}\left(\left\{\gamma_{1}^{\text {OWFD }}<\tau\right\} \cap\left\{\gamma_{2}^{\text {OWFD }}<\tau\right\}\right)}_{P_{\text {out }, 12}},
\end{aligned}
$$

where

$$
\begin{aligned}
& P_{\text {out }, 12}^{\text {OWFD }}=\operatorname{Pr}\left(\left\{\gamma_{1}^{\text {OWFD }}<\tau\right\} \cap\left\{\gamma_{2}^{\text {OWFD }}<\tau\right\}\right) \\
& =\operatorname{Pr}\left(\left\{\frac{a_{2} x y}{b x+c_{2} x y+c}<\tau\right\} \cap\left\{\frac{a_{1} x y}{b y+c_{1} x y+c}<\tau\right\}\right) \\
& =\operatorname{Pr}\left(\left\{y<\frac{\tau(b x+c)}{\left(a_{2}-\tau c_{2}\right) x}\right\} \cap\left\{x<\frac{\tau(b y+c)}{\left(a_{1}-\tau c_{1}\right) y}\right\}\right) \\
& =\operatorname{Pr}\left(\left\{y<\frac{\tau(b x+c)}{a x}\right\} \cap\left\{x<\frac{\tau(b y+c)}{d y}\right\}\right) \\
& =\mathcal{P}_{1}+\mathcal{P}_{2}
\end{aligned}
$$

and

$$
\begin{aligned}
\mathcal{P}_{1} & =-\lambda_{1} e^{-\frac{\lambda_{2} b \tau}{a}}\left(\sqrt{\frac{\beta_{1}}{\lambda_{1}}} K_{1}\left(\sqrt{\beta_{1} \lambda_{1}}\right)\right. \\
& \left.-\sum_{t=0}^{\infty} \frac{(-1)^{t} \phi_{1}^{t}}{t !}\left(x_{0}\right)^{1-t} E_{t}\left(\lambda_{1} x_{0}\right)\right) \\
& -\frac{\lambda_{1}}{\lambda_{2} y_{0} / x_{0}+\lambda_{1}}\left(e^{-\left(\lambda_{2} y_{0}+\lambda_{1} x_{0}\right)}-1\right)
\end{aligned}
$$

and

$$
\begin{aligned}
\mathcal{P}_{2} & =-\lambda_{2} e^{-\frac{\lambda_{1} b \tau}{d}}\left(\sqrt{\frac{\beta_{2}}{\lambda_{2}}} K_{1}\left(\sqrt{\beta_{2} \lambda_{2}}\right)\right. \\
& \left.-\sum_{t=0}^{\infty} \frac{(-1)^{t} \phi_{2}^{t}}{t !}\left(y_{0}\right)^{1-t} E_{t}\left(\lambda_{2} y_{0}\right)\right) \\
& -\frac{\lambda_{2}}{\lambda_{1} x_{0} / y_{0}+\lambda_{2}}\left(e^{-\left(\lambda_{1} x_{0}+\lambda_{2} y_{0}\right)}-1\right)
\end{aligned}
$$

Replacing (30) and (31) into (29), we obtain $P_{\text {out }, 12}^{O W F D}$. Finally, we achieve the closed-form expression of the end-to-end overall OP as

$$
\begin{aligned}
P_{e 2 e}^{O W F D} & =1-\lambda_{1} e^{-\frac{\lambda_{2} \tau b_{2}}{\left(a_{2}-\tau c_{2}\right)}} \sqrt{\frac{\psi}{\lambda_{1}}} K_{1}\left(\sqrt{\psi \lambda_{1}}\right) \\
& +1-\lambda_{2} e^{-\frac{\lambda_{1} \tau b_{1}}{\left(a_{1}-\tau c_{1}\right)}} \sqrt{\frac{\vartheta}{\lambda_{2}}} K_{1}\left(\sqrt{\vartheta \lambda_{2}}\right) \\
& +\lambda_{1} e^{-\frac{\lambda_{2} b \tau}{a}}\left(\sqrt{\frac{\beta_{1}}{\lambda_{1}}} K_{1}\left(\sqrt{\beta_{1} \lambda_{1}}\right)\right. \\
& \left.-\sum_{t=0}^{\infty} \frac{(-1)^{t} \phi_{1}{ }^{t}}{t !}\left(x_{0}\right)^{1-t} E_{t}\left(\lambda_{1} x_{0}\right)\right) \\
& +\frac{\lambda_{1}}{\lambda_{2} y_{0} / x_{0}+\lambda_{1}}\left(e^{-\left(\lambda_{2} y_{0}+\lambda_{1} x_{0}\right)}-1\right) \\
& +\lambda_{2} e^{-\frac{\lambda_{1} b \tau}{d}}\left(\sqrt{\frac{\beta_{2}}{\lambda_{2}}} K_{1}\left(\sqrt{\beta_{2} \lambda_{2}}\right)\right. \\
& \left.-\sum_{t=0}^{\infty} \frac{(-1)^{t} \phi_{2}{ }^{t}}{t !}\left(y_{0}\right)^{1-t} E_{t}\left(\lambda_{2} y_{0}\right)\right) \\
& +\frac{\lambda_{2}}{\lambda_{1} x_{0} / y_{0}+\lambda_{2}}\left(e^{-\left(\lambda_{1} x_{0}+\lambda_{2} y_{0}\right)}-1\right),
\end{aligned}
$$

where $a=a_{2}-\tau c_{2}, d=a_{1}-\tau c_{1}, x_{0}=\frac{\varphi+\sqrt{\varphi^{2}+4 \tau a b^{2} c}}{2 a b}$, $y_{0}=\frac{\tau\left(b x_{0}+c\right)}{a x_{0}}, \varphi=-a c+\tau b^{2}+c d$.

Please see Appendix D for detailed derivation of (32).

\subsection{The OP of the TWFD Communications}

The individual OP is defined as

$$
P_{\text {out }, i}^{T W F D}=\operatorname{Pr}\left(\gamma_{i}^{T W F D}<\tau\right) .
$$

The throughput of the TWFD communications is given by

$$
T_{0}=R_{T}\left(1-P_{o u t, i}^{T W F D}\right)(1-\alpha) .
$$

$$
P_{\text {out }, 1}^{T W F D} \text { is computed as }
$$

$$
\begin{aligned}
P_{\text {out }, 1}^{T W F D} & =\operatorname{Pr}\left(\gamma_{1}^{T W F D}<\tau\right) \\
& =\operatorname{Pr}\left(\frac{e_{1} x y}{x\left[f_{1}\left(P_{1} x+P_{2} y+g_{1}\right)+g_{1}\right]+k_{1}}<\tau\right) .
\end{aligned}
$$

Perform further simplifications, we have

$$
\begin{aligned}
& P_{\text {out }, 1}^{T W F D}= \\
& \left\{\begin{array}{cc}
\operatorname{Pr}\left(y<\frac{\tau x\left(f_{1} P_{1} x+f_{1} g_{1}+g_{1}\right)+\tau k_{1}}{x\left(e_{1}-\tau f_{1} P_{2}\right)}\right) & , e_{1}-\tau f_{1} P_{2}>0 \\
1 & , e_{1}-\tau f_{1} P_{2}<0
\end{array}\right.
\end{aligned}
$$

As shown in Appendix C, $P_{\text {out }, 1}^{T W F D}$ in (36) can be represented in the closed form for the case of $e_{1}-\tau f_{1} P_{2}>0$ as

$$
P_{\text {out }, 1}^{T W F D}=1-\lambda_{1} e^{-\frac{\lambda_{2} \tau\left(f_{1} g_{1}+g_{1}\right)}{e_{1}-\tau f_{1} P_{2}}} \sqrt{\frac{\Omega_{1}}{\Psi_{1}}} K_{1}\left(\sqrt{\Omega_{1} \Psi_{1}}\right),
$$

where $\Omega_{1}=\frac{4 \lambda_{2} \tau k_{1}}{\left(e_{1}-\tau f_{1} P_{2}\right)}$ and $\Psi_{1}=\frac{\lambda_{2} \tau f_{1} P_{1}}{e_{1}-\tau f_{1} P_{2}}+\lambda_{1}$. 
Following the same approach as (37), we have

$$
\begin{aligned}
P_{\text {out }, 2}^{T W F D} & =\operatorname{Pr}\left(\gamma_{2}^{T W F D}<\tau\right) \\
& =1-\lambda_{2} e^{-\frac{\lambda_{1} \tau\left(f_{2} g_{2}+g_{2}\right)}{e_{2}-\tau f_{2} P_{1}}} \sqrt{\frac{\Omega_{2}}{\Psi_{2}}} K_{1}\left(\sqrt{\Omega_{2} \Psi_{2}}\right),
\end{aligned}
$$

where $\Omega_{2}=\frac{4 \lambda_{1} \tau k_{2}}{\left(e_{2}-\tau f_{2} P_{1}\right)}$ and $\Psi_{2}=\frac{\lambda_{1} \tau f_{2} P_{2}}{e_{2}-\tau f_{2} P_{1}}+\lambda_{2}$.

Please see Appendix C for detailed derivation of (38).

The end-to-end overall OP of the AF based TWFD communications is defined as [9, Eq. (9)]

$$
\begin{aligned}
P_{e 2 e}^{T W F D} & =\operatorname{Pr}\left(\min \left(\gamma_{1}^{T W F D}, \gamma_{2}^{T W F D}\right)<\tau\right) \\
& =1-\operatorname{Pr}\left(\gamma_{1}^{T W F D}>\tau, \gamma_{2}^{T W F D}>\tau\right) .
\end{aligned}
$$

From (39), we have

$$
\begin{aligned}
P_{e 2 e}^{T W F D} & =\underbrace{\operatorname{Pr}\left(\gamma_{1}^{T W F D}<\tau\right)}_{P_{\text {out } t 1}^{T W F D}}+\underbrace{\operatorname{Pr}\left(\gamma_{2}^{T W F D}<\tau\right)}_{P_{\text {out }, 2}^{T W F D}} \\
- & \underbrace{\operatorname{Pr}\left(\left\{\gamma_{1}^{T W F D}<\tau\right\} \cap\left\{\gamma_{2}^{T W F D}<\tau\right\}\right)}_{P_{\text {out }, 12}^{\text {TWFD }}},
\end{aligned}
$$

where $P_{\text {out }, 1}^{T W F D}$ and $P_{\text {out }, 2}^{T W F D}$ are given in (37) and (38), respectively.

We approximate the component $P_{\text {out }, 12}^{T W F D}$ in (40) as

$$
\begin{aligned}
P_{\text {out }, 12}^{T W F D} & =\operatorname{Pr}\left(\left\{\gamma_{1}^{\text {TWFD }}<\tau\right\} \cap\left\{\gamma_{2}^{\text {TWFD }}<\tau\right\}\right) \\
& \simeq \operatorname{Pr}\left(\left\{\gamma_{11}<\tau\right\} \cap\left\{\gamma_{22}<\tau\right\}\right),
\end{aligned}
$$

where $\gamma_{11}$ and $\gamma_{22}$ are given by

$$
\begin{aligned}
\gamma_{1}^{T W F D} & =\frac{e_{1} x y}{x\left[f_{1}\left(P_{1} x+P_{2} y+g_{1}\right)+g_{1}\right]+k_{1}} \\
& =\frac{e_{1} x y}{f_{1} P_{1} x^{2}+f_{1} P_{2} x y+f_{1} g_{1} x+g_{1} x+k_{1}} \\
& \leq \frac{e_{1} x y}{f_{1} P_{1} x+f_{1} P_{2} x y+f_{1} g_{1} x+g_{1} x+k_{1}} \\
& =\frac{e_{1} x y}{\left(f_{1} P_{1}+f_{1} g_{1}+g_{1}\right) x+f_{1} P_{2} x y+k_{1}} \\
& =\gamma_{11},
\end{aligned}
$$

and

$$
\begin{aligned}
\gamma_{2}^{T W F D} & =\frac{e_{2} x y}{y\left[f_{2}\left(P_{1} x+P_{2} y+g_{2}\right)+g_{2}\right]+k_{2}} \\
& =\frac{e_{2} x y}{f_{2} P_{1} x y+f_{2} P_{2} y^{2}+f_{2} g_{2} y+y g_{2}+k_{2}} \\
& \leq \frac{e_{2} x y}{f_{2} P_{1} x y+f_{2} P_{2} y+f_{2} g_{2} y+y g_{2}+k_{2}} \\
& =\frac{e_{2} x y}{\left(f_{2} P_{2}+f_{2} g_{2}+g_{2}\right) y+f_{2} P_{1} x y+k_{2}} \\
& =\gamma_{22} .
\end{aligned}
$$

It is noted that approximations in (42) and (43) are valid because $x$ and $y$ are channel gains, i.e., $0<x$, $y<1$.

Without loss of generality, for performance comparison between the TWFD and OWFD schemes, we choose $P=P_{1}=P_{2}$. Therefore, the approximated SNRs in (42) and (43) are similar to those of the OWFD, i.e.,

$$
\begin{aligned}
\gamma_{11} & =\frac{e_{1} x y}{\left(f_{1} P_{1}+f_{1} g_{1}+g_{1}\right) x+f_{1} P_{2} x y+k_{1}} \\
& =\frac{e_{1} x y}{B x+C_{1} x y+C}
\end{aligned}
$$

and

$$
\begin{aligned}
\gamma_{22} & =\frac{e_{2} x y}{f_{2} P_{1} x y+\left(f_{2} P_{2}+f_{2} g_{2}+g_{2}\right) y+k_{2}} \\
& =\frac{e_{2} x y}{B y+C_{2} x y+C^{\prime}}
\end{aligned}
$$

where $B \triangleq f_{1} P_{1}+f_{1} g_{1}+g_{1} \triangleq f_{2} P_{2}+f_{2} g_{2}+g_{2}, C \triangleq$ $k_{1} \triangleq k_{2}, C_{1}=f_{1} P_{2}, C_{2}=f_{2} P_{2}$.

Remaining parameters as defined in (21) and (22), we have from (41):

$$
\begin{aligned}
P_{\text {out }, 12}^{T W F D} & \simeq-\lambda_{1} e^{-\frac{\lambda_{2} b \tau}{a}}\left(\sqrt{\frac{\beta_{1}}{\lambda_{1}}} K_{1}\left(\sqrt{\beta_{1} \lambda_{1}}\right)\right. \\
& \left.-\sum_{t=0}^{\infty} \frac{(-1)^{t} \phi_{1}^{t}}{t !}\left(x_{0}\right)^{1-t} E_{t}\left(\lambda_{1} x_{0}\right)\right) \\
& -\frac{\lambda_{1}}{\lambda_{2} y_{0} / x_{0}+\lambda_{1}}\left(e^{-\left(\lambda_{2} y_{0}+\lambda_{1} x_{0}\right)}-1\right) \\
& -\lambda_{2} e^{-\frac{\lambda_{1} b \tau}{d}}\left(\sqrt{\frac{\beta_{2}}{\lambda_{2}}} K_{1}\left(\sqrt{\beta_{2} \lambda_{2}}\right)\right. \\
& \left.-\sum_{t=0}^{\infty} \frac{(-1)^{t} \phi_{2}^{t}}{t !}\left(y_{0}\right)^{1-t} E_{t}\left(\lambda_{2} y_{0}\right)\right) \\
& -\frac{\lambda_{2}}{\lambda_{1} x_{0} / y_{0}+\lambda_{2}}\left(e^{-\left(\lambda_{1} x_{0}+\lambda_{2} y_{0}\right)}-1\right),
\end{aligned}
$$

where $a=e_{1}-\tau C_{1}$, and $d=e_{2}-\tau C_{2}, x_{0}=$ $\frac{\varphi+\sqrt{\varphi^{2}+4 \tau a b^{2} c}}{2 a b}, y_{0}=\frac{\tau\left(b x_{0}+c\right)}{a x_{0}}, \varphi=-a c+\tau b^{2}+c d$.

Please see Appendix D for detailed derivation of (46).

Replacing (37), (38) and (46) into (40), we obtain the approximate closed-form OP formula for the TWFD communications.

\section{Simulation Results}

In this section, the simulation results are presented to evaluate the performance of the OWFD and the TWFD communications as well as to compare them with the TWHD communications. The effect of the SI on the $\mathrm{OP}$ is evaluated via key parameters such as SNR, the time switching ratio $\alpha$, the energy conversion efficiency $\beta$, the target transmission rate $R_{T}$, the transmit power of each source. Toward this end, we choose the coordinates of $S_{1}$ at $(0.0,0.0)$, and $S_{2}$ at $(1.0,0.0)$, and $R$ at $(0.5,0.0)$. For demonstration purpose, the same transmit powers are considered, i.e., $P_{1}=P_{2}=P$. The SI at all the nodes are assumed to be the same, i.e., $\sigma_{11}^{2}=\sigma_{22}^{2}=\sigma_{r r}^{2}=\sigma_{S I}^{2}=S I$. The path-loss exponent is fixed at $\chi=3$. In the following figures, "The." and "Sim." represent the analytical and the simulated results, respectively.

Figure 3 shows the throughput of TWFD, OWFD and TWHD with $\beta=0.5, R_{T}=1 \mathrm{bps} / \mathrm{Hz}, \sigma_{S I}^{2}=1$ for two cases of $\alpha=0.2$ and $\alpha=0.5$. The throughput of 


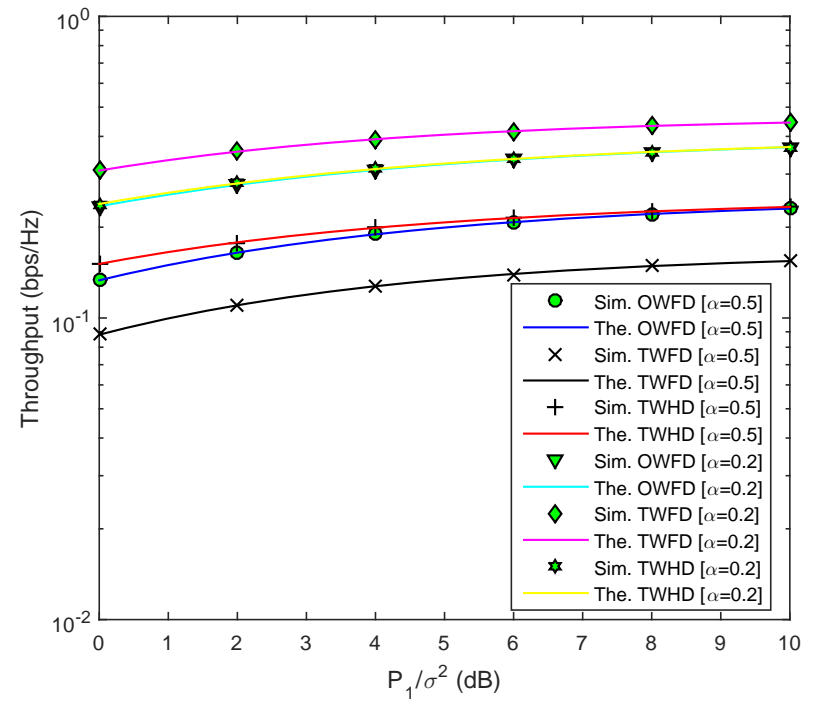

Figure 3. Throughput for TWFD, OWFD and TWHD.

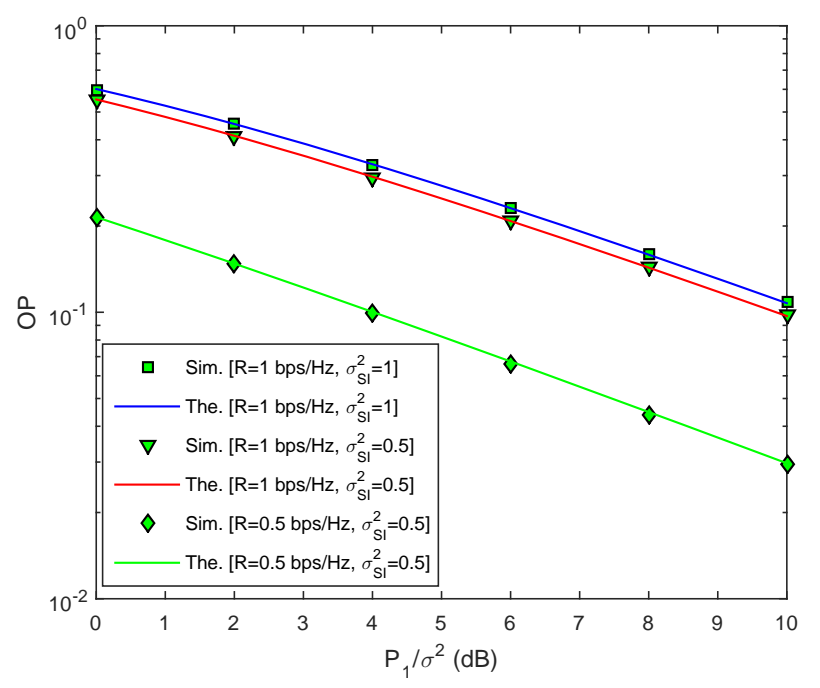

Figure 4. OP of the OWFD via SNR.

the OWFD is obtained from (24) while the throughput of the TWFD is obtained from (34). However, the throughput of the TWHD is achieved from (24) with $\sigma_{S I}^{2}=0$. The results show that the theoretical analysis matches well with the Monte-Carlo simulation. Also, the throughput is increased with higher SNR because the OP decreases in (24) and (34). Moreover, when $\alpha$ is small, the throughput of the TWHD and the OWFD are greater than that of the TWFD. This can be explained from the fact that the SI affects the TWFD more than the OWFD and the TWHD. Furthermore, when $\alpha$ increases, the remaining time for information processing decreases; therefore, the TWFD only needs one timeslot for information processing while the TWHD and the OWFD need two time-slots for signal processing. This improves the throughput of the TWFD.

Figure 4 evaluates to the effect of the SI on the OP of the OWFD. The simulation parameters are $\alpha=\beta=0.5$, two cases of $R_{T}=0.5 \mathrm{bps} / \mathrm{Hz}$ and $R_{T}=1 \mathrm{bps} / \mathrm{Hz}$, $\sigma_{S I}^{2}=0.5$, and $\sigma_{S I}^{2}=1$. It is seen that the simulated results match well with the theoretical ones, verifying

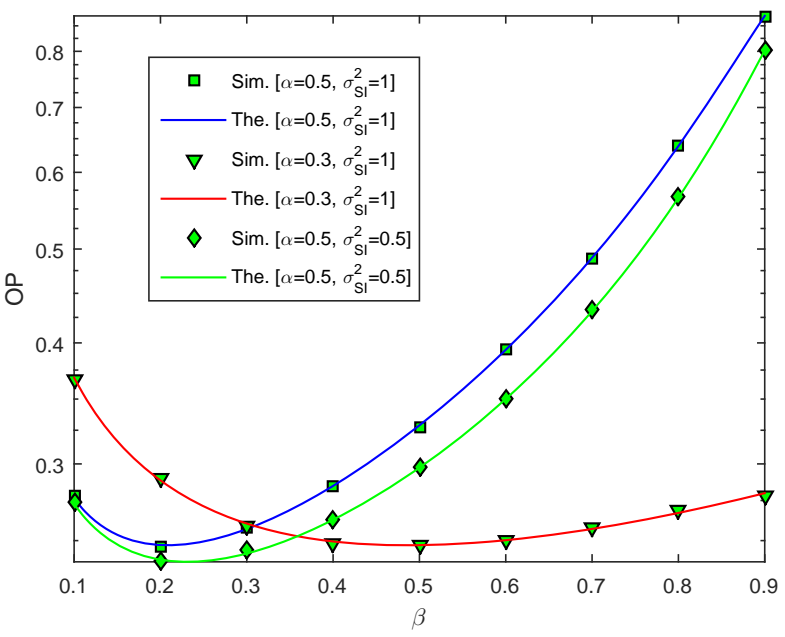

Figure 5. OP of the OWFD via $\beta$.

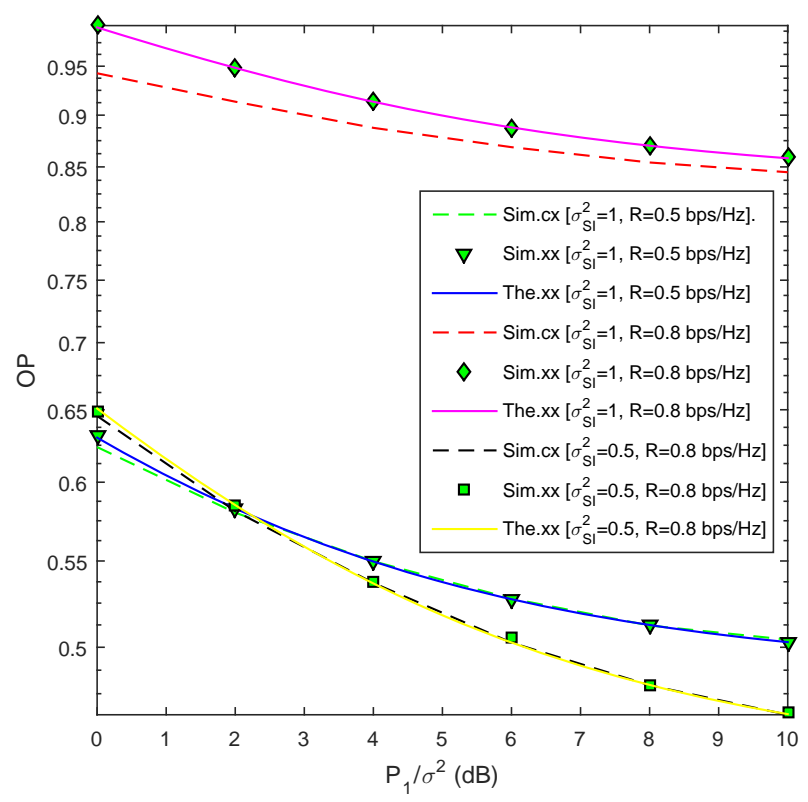

Figure 6. OP of the TWFD via SNR.

the exactness of the proposed closed-form overall OP in (28). Moreover, when the SNR increases, the performance is improved because the outage gets lower. Furthermore, the OP increases due to the effect of the SI because higher SI, the lower SNR is. For the same SI level, the OP increases with higher fixed transmission rate. This is because the higher fixed transmission rate requires the higher throughput; therefore, the same SNR causes more outage for the system.

The parameters in Figure 5 are $P_{1}=P_{2}=4 \mathrm{~dB}, R_{T}=$ $1 \mathrm{bps} / \mathrm{Hz}$, two cases of $\alpha=0.3$ and $\alpha=0.5, \sigma_{S I}^{2}=0.5$ and $\sigma_{S I}^{2}=1$. It is observed that the SI affects the OP of the OWFD; the higher the SI is, the larger OP is. Additionally, the OP decreases when $\alpha$ is smaller. This can be explained from the fact that the OWFD can have more time for signal processing, improving the system performance. Furthermore, there is an optimum value of $\alpha$ and $\beta$ for the minimum OP.

In Figure 6, we simulate with the parameters: $\alpha=$ 


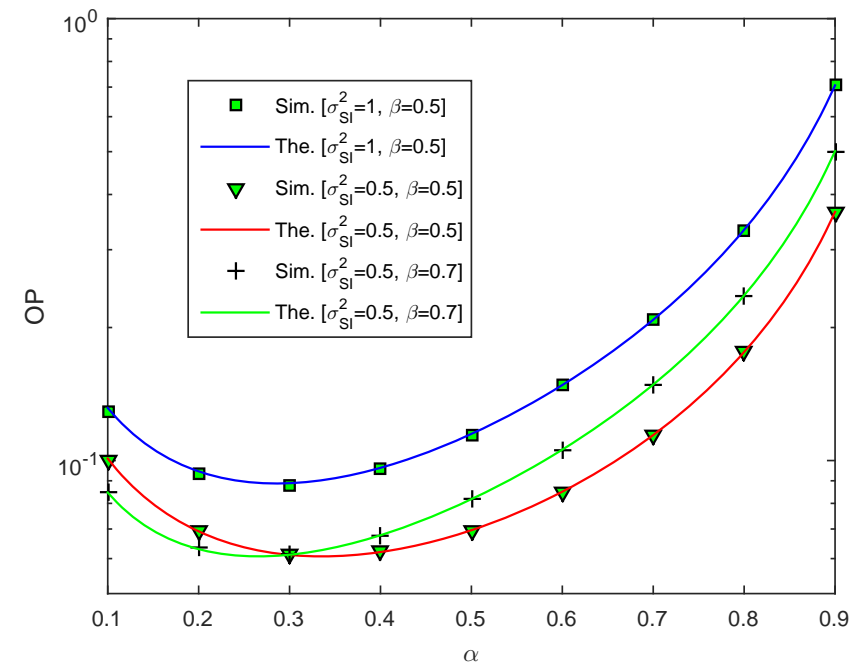

Figure 7. OP of the TWFD via $\alpha$

$\beta=0.5$, two cases of $\sigma_{S I}^{2}=0.5$ and $\sigma_{S I}^{2}=1, R_{T}=0.5$ $\mathrm{bpz} / \mathrm{Hz}$ and $R_{T}=0.8 \mathrm{bps} / \mathrm{Hz}$. In this figure, "Sim.cx" represented by dash lines is the exact simulation of $P_{\text {out }, 12}^{T W F D}$ in (40) while "Sim.xx" and "The.xx" are the simulation and the theory of the approximate $P_{\text {out }, 12}^{T W F D}$ in (41). It is clear that the SI affects significantly the OP performance. Moreover, the higher SI is, the larger OP is. For higher SNRs, the exact OP bound coincides the approximate OP. Furthermore, at the lower fixed transmission rate, the "Sim.xx" line is close to "Sim.cx" line as illustrated in (42) and (43).

Figure 7 shows the effect of $\alpha$ on the OP of the TWFD communications for $P_{1}=P_{2}=2 \mathrm{~dB}, R_{T}=0.1 \mathrm{bps} / \mathrm{Hz}$, two cases of $\beta=0.5$ and $\beta=0.7$, and $\sigma_{S I}^{2}=0.5$ and $\sigma_{S I}^{2}=1$. The simulation results matched well analysis results. This figure shows that for the same $\beta$, the OP increases when the SI increases because the TWFD uses all the nodes which work in full-duplex mode; hence, they suffer more SI, resulting in the lower end-to-end SNR. Further, when the $\beta$ is small, the energy efficiency gets lower; so, the relay node has no enough energy to forward the information, causing system outage. When the $\alpha$ is higher, the OP is higher. This can be explained as follows. Although the relay node can harvest more energy, the remaining time for signal processing also decreases; so, the OP increases.

The parameters in Figure 8 are $\alpha=\beta=0.5, R_{T}=0.5$ $\mathrm{bps} / \mathrm{Hz}$, two cases of $\sigma_{S I}^{2}=0.5$ and $\sigma_{S I}^{2}=1$. This figure shows that the OP of the TWFD is greater than those of the OWFD and the TWHD. This is explained from the fact that the TWFD suffers more SI than the OWFD. For the TWHD, the SI is zero. As such, to improve the performance of the TWFD and the OWFD, the SI needs to be removed or minimized. It is seen that the analysis exactly agrees the simulation, verifying the precision of the proposed analysis. Additionally, the outage probability is inversely proportional to the SNR.

The parameters in Figure 9 are $\alpha=0.5, R_{T}=0.5$ bps $/ \mathrm{Hz}, P_{1}=P_{2}=4 \mathrm{~dB}$, two cases of $\sigma_{S I}^{2}=0.5$ and $\sigma_{S I}^{2}=1$. It is observed that the OP of the TWFD and the OWFD increases quickly with higher SI level.

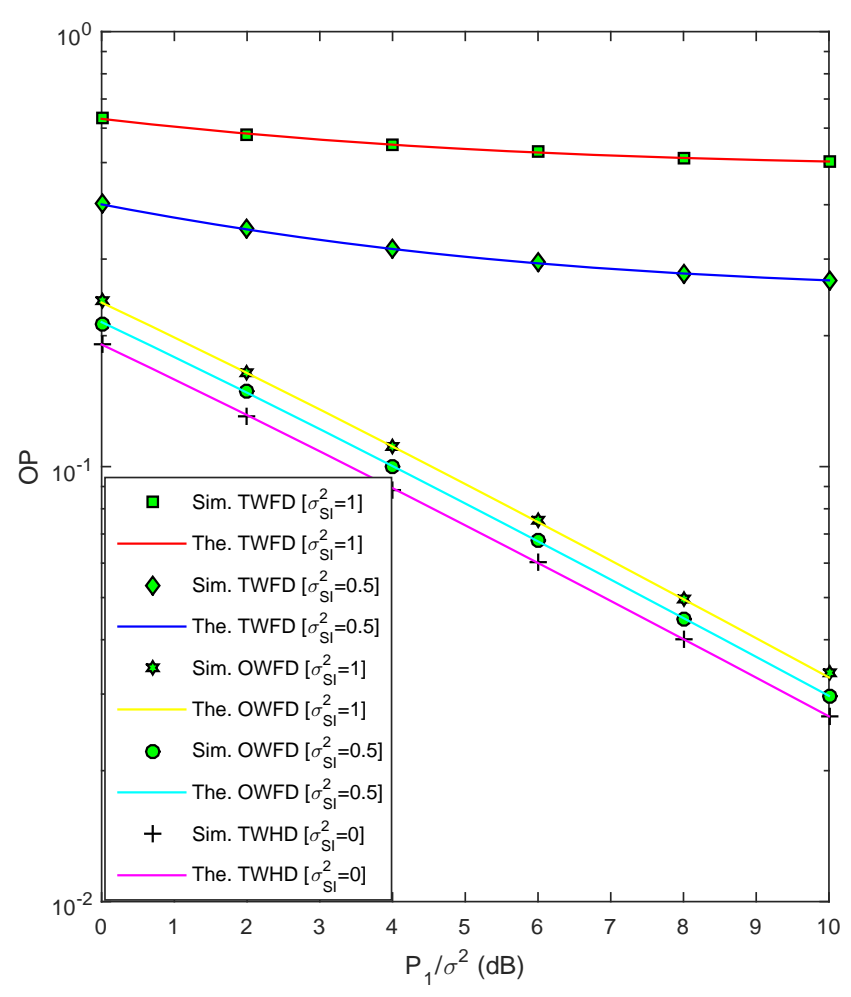

Figure 8. OP via SNR

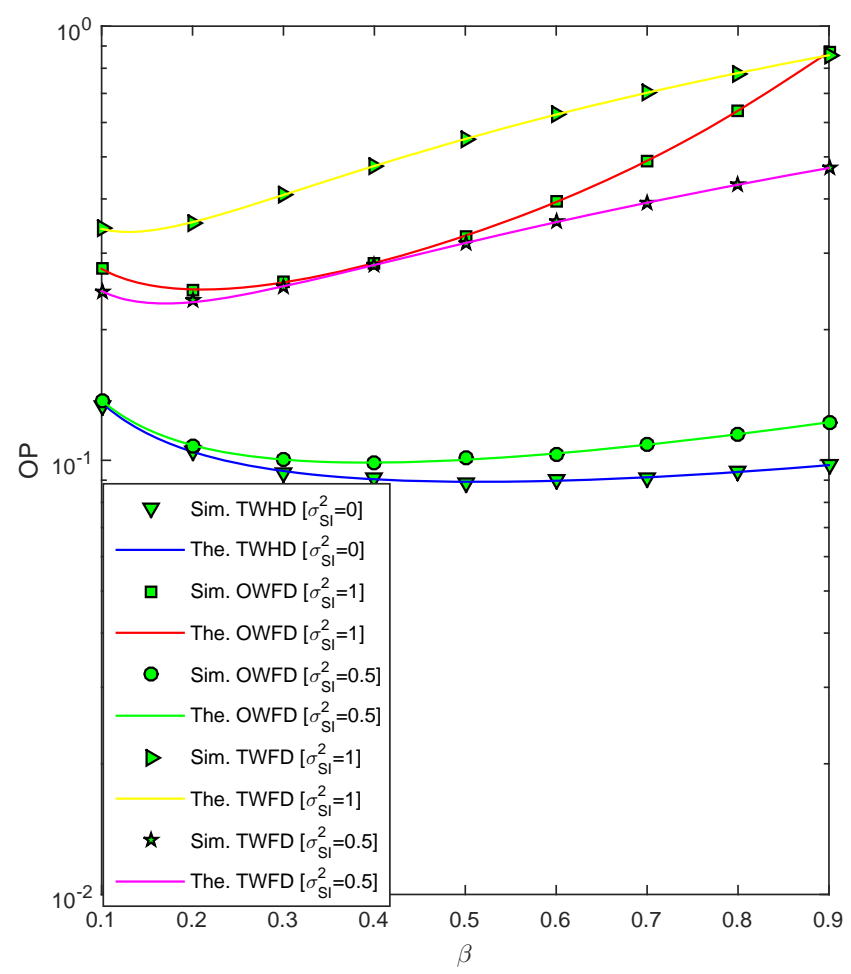

Figure 9. OP via $\beta$.

Furthermore, the OP of the TWHD is the least because it is not affected by the SI. Moreover, because the TWFD uses all nodes with FD while the OWFD has only one FD at the relay. It is inevitable that the TWFD suffers more severe residual self-interference than the OWFD and the TWHD.

The parameters in Figure 10 are $\beta=0.5, R_{T}=0.1$ 


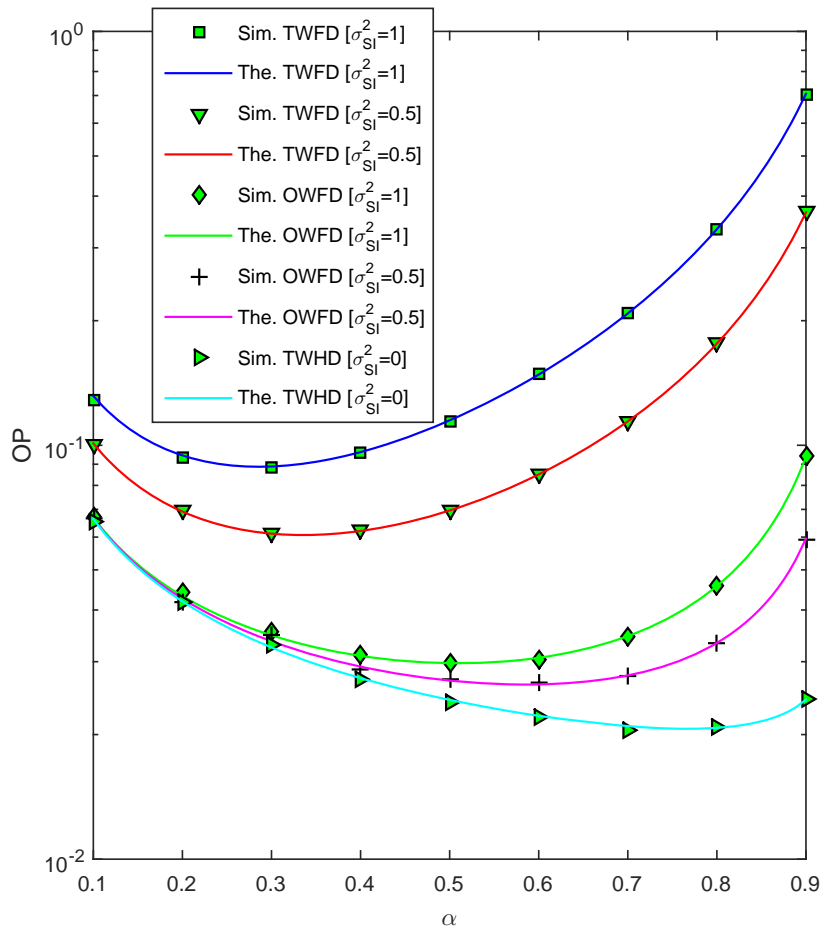

Figure 10. OP via $\alpha$.

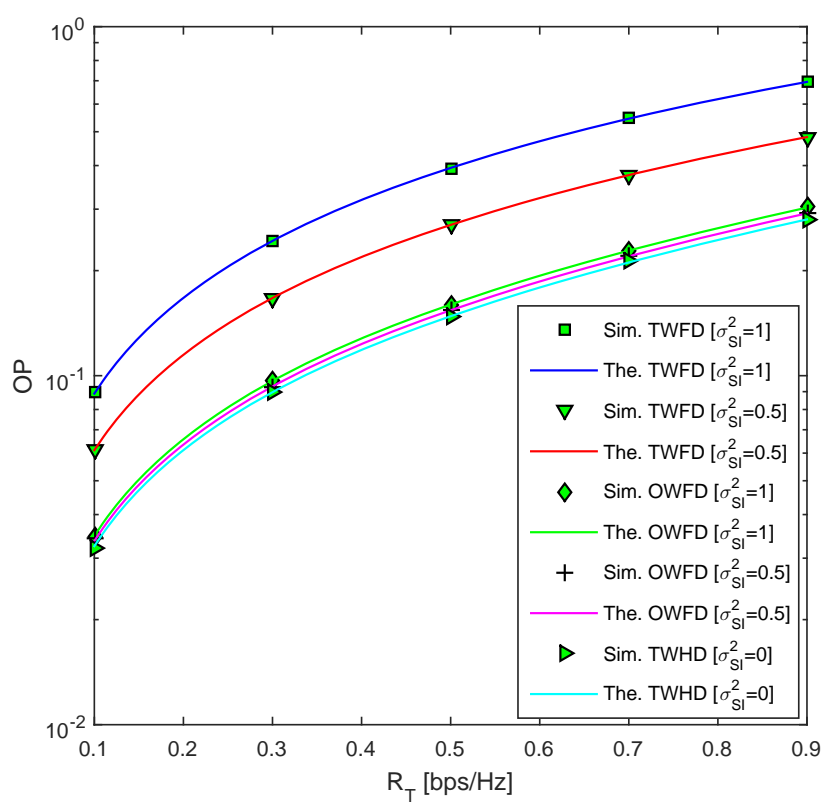

Figure 11. OP via fixed transmission rate.

bps $/ \mathrm{Hz}, P_{1}=P_{2}=2 \mathrm{~dB}$, two cases of $\sigma_{S I}^{2}=0.5$ and $\sigma_{S I}^{2}=1$. The results show that the lower the SI is, the lower the OP is. Moreover, for the same SI level, the OP of the TWFD is greater than that of the OWFD. This can be explained from the fact that the TWFD uses all nodes suffering more SI while the TWHD is not affected by the SI; therefore, the OP of the TWHD is the least. Furthermore, with the lower SI level, the OP of the OWFD is close to that of the TWHD.

The parameters in Figure 11 are $\beta=0.5, \alpha=0.3$, $P_{1}=P_{2}=2 \mathrm{~dB}$, two cases of $\sigma_{S I}^{2}=0.5$ and $\sigma_{S I}^{2}=1$. It is observed that the OP increases at higher trans- mission rate because the higher required transmission rate needs the higher SNR for the same OP. This figure also shows the exact agreement between the analysis and the simulation. Moreover, the outage probability is proportional to the transmission rate. This can be explained by the fact that higher demand on target transmission rates induces the system unable to satisfy, causing higher outage probability.

\section{Conclusions}

This paper presented an efficient method to calculate the overall $\mathrm{OP}$ of the AF relaying systems with the TWFD and the OWFD. Their OP was also compared with that of the TWHD. The simulated results validated the proposed method. Moreover, the TWFD and the OWFD communications are considerably deteriorated by the self-interference due to the FD operation at the EH relay. Therefore, the SI cancellation techniques should be further exploited to improve the system performance for the TWFD and the OWFD communications, which is our future work.

\section{ACKNOWLEDGMENTS}

This research is funded by Ho Chi Minh City University of Technology - VNU-HCM under grant number TNCSĐĐT-2017-03.

\section{Appendix A}

This section will prove the expressions of the SNRs in (9) and (10). From (8), we have

$$
\begin{aligned}
& \gamma_{2}^{O W F D}=\frac{E\left\{\mid \text { signal }\left.\right|^{2}\right\}}{E\left\{\mid \text { noise }\left.\right|^{2}\right\}} \\
& =\frac{\theta_{1}^{2} P_{R} P_{1}\left|h_{1}\right|^{2}\left|h_{2}\right|^{2} E\left\{\left|x_{1}[t-1]\right|^{2}\right\}}{\theta_{1}^{2} P_{R}\left|h_{2}\right|^{2}\left|h_{r r}\right|^{2} E\left\{\left|x_{R}[t-1]\right|^{2}\right\}+\theta_{1}^{2} P_{R}\left|h_{2}\right|^{2} \sigma_{R}^{2}+\sigma_{2}^{2}} \\
& =\frac{\theta_{1}^{2} P_{R} P_{1}\left|h_{1}\right|^{2}\left|h_{2}\right|^{2}}{\theta_{1}{ }^{2}\left(P_{R}\right)^{2}\left|h_{2}\right|^{2} \sigma_{S I}^{2}+\theta_{1}{ }^{2} P_{R}\left|h_{2}\right|^{2} \sigma^{2}+\sigma^{2}} .
\end{aligned}
$$

Replacing $P_{R}$ in (2) and $\theta_{1}$ in (5) into (47), we have

$$
\begin{aligned}
\gamma_{2}^{O W F D} & =\frac{P_{1}\left|h_{1}\right|^{2}\left|h_{2}\right|^{2}}{P_{R}\left|h_{2}\right|^{2} \sigma_{S I}^{2}+\left|h_{2}\right|^{2} \sigma^{2}+\frac{\sigma^{2}}{P_{R} \theta_{1}{ }^{2}}} \\
& =\frac{P_{1}\left|h_{1}\right|^{2}\left|h_{2}\right|^{2}}{\left|h_{2}\right|^{2} \sigma^{2}+P_{R}\left|h_{2}\right|^{2} \sigma_{S I}^{2}+\frac{\sigma^{2}\left(P_{1}\left|h_{1}\right|^{2}+P_{R}\left|h_{r r}\right|^{2}+\sigma^{2}\right)}{P_{R}}} \\
& =\frac{P_{1}\left|h_{1}\right|^{2}\left|h_{2}\right|^{2}}{\left|h_{2}\right|^{2} \sigma^{2}+P_{R}\left|h_{2}\right|^{2} \sigma_{S I}^{2}+\frac{\sigma^{2}\left(\frac{P_{R}}{\phi}+P_{R}\left|h_{r r}\right|^{2}\right)}{P_{R}}} \\
& =\frac{P_{1}\left|h_{1}\right|^{2}\left|h_{2}\right|^{2}}{\left|h_{2}\right|^{2} \sigma^{2}+P_{R}\left|h_{2}\right|^{2} \sigma_{S I}^{2}+\sigma^{2}\left(\frac{1}{\phi}+\left|h_{r r}\right|^{2}\right)} .
\end{aligned}
$$


From (48), we have

$\gamma_{2}^{O W F D}=$

$\frac{P_{1}\left|h_{1}\right|^{2}\left|h_{2}\right|^{2}}{\left|h_{2}\right|^{2} \sigma^{2}+\phi P_{1}\left|h_{1}\right|^{2}\left|h_{2}\right|^{2} \sigma_{S I}^{2}+\phi\left|h_{2}\right|^{2} \sigma_{S I}^{2} \sigma^{2}+\sigma^{2}\left(\frac{1}{\phi}+\left|h_{r r}\right|^{2}\right)}$.

After some manipulations, we have $\gamma_{2}^{O W F D}$

$$
\begin{aligned}
& =\frac{P_{1}\left|h_{1}\right|^{2}\left|h_{2}\right|^{2}}{\left|h_{2}\right|^{2}\left(\sigma^{2}+\phi \sigma_{S I}^{2} \sigma^{2}\right)+\phi P_{1}\left|h_{1}\right|^{2}\left|h_{2}\right|^{2} \sigma_{S I}^{2}+\sigma^{2}\left(\frac{1}{\phi}+\sigma_{S I}^{2}\right)} \\
& =\frac{a_{1} x y}{b y+c_{1} x y+c},
\end{aligned}
$$

where $E\left\{\left|h_{r r}\right|^{2}\right\}=\sigma_{r r}^{2}=\sigma_{S I}^{2}$

This completes the proof of (9).

The same approach for $S_{2} \rightarrow R \rightarrow S_{1}$, we have

$$
\theta_{2}=\frac{1}{\sqrt{P_{2}\left|h_{2}\right|^{2}+P_{R}\left|h_{r r}\right|^{2}+\sigma^{2}}} .
$$

The received signal at $S_{1}$ is

$$
y_{1}[t]=h_{1} x_{R}[t]+n_{1}[t],
$$

where

$$
x_{R}[t]=\sqrt{P_{R}} \theta_{2} y_{R}[t-1] .
$$

Replacing (51) and (52) into (53), we obtain

$$
\begin{aligned}
& y_{1}[t]=\theta_{2} \sqrt{P_{R}} h_{1} \times \\
& \left(\sqrt{P_{2}} h_{2} x_{2}[t-1]+h_{r r} x_{R}[t-1]+n_{R}[t-1]\right)+n_{1}[t] \\
& =\underbrace{\theta_{2} \sqrt{P_{R}} \sqrt{P_{2}} h_{1} h_{2} x_{2}[t-1]}_{\text {signal }}+\underbrace{\theta_{2} \sqrt{P_{R}} h_{1} h_{r r} x_{R}[t-1]}_{\text {SI }} \\
& +\underbrace{\theta_{2} \sqrt{P_{R}} h_{1} n_{R}[t-1]+n_{1}[t]}_{\text {noise }}
\end{aligned}
$$

From (54), one infers the SNR at $S_{1}$ as $\gamma_{1}^{O W F D}=$

$$
\begin{aligned}
& \frac{P_{2}\left|h_{1}\right|^{2}\left|h_{2}\right|^{2}}{\left|h_{1}\right|^{2}\left(\sigma^{2}+\phi \sigma_{S I}^{2} \sigma^{2}\right)+\phi P_{2}\left|h_{1}\right|^{2}\left|h_{2}\right|^{2} \sigma_{S I}^{2}+\sigma^{2}\left(\frac{1}{\phi}+\sigma_{S I}^{2}\right)} \\
& =\frac{a_{2} x y}{b x+c_{2} x y+c},
\end{aligned}
$$

which completes the proof of (10).

\section{Appendix B}

This section will prove the expressions of the SNRs in (21) and (22). From (19), we have (56).

We further simplify (56) as

$$
\begin{aligned}
& \gamma_{1}^{T W F D}= \\
& \frac{P_{2}\left|h_{1}\right|^{2}\left|h_{2}\right|^{2}}{\left|h_{1}\right|^{2}\left|h_{r r}\right|^{2} \phi\left(P_{1}\left|h_{1}\right|^{2}+P_{2}\left|h_{2}\right|^{2}+\sigma_{R}^{2}\right)+\left|h_{1}\right|^{2} \sigma_{R}^{2}+k_{1}} .
\end{aligned}
$$

After some simplifications, we have $\gamma_{1}^{T W F D}=$

$\frac{P_{2}\left|h_{1}\right|^{2}\left|h_{2}\right|^{2}}{\left|h_{1}\right|^{2}\left\{\sigma_{S I}^{2} \phi\left(P_{1}\left|h_{1}\right|^{2}+P_{2}\left|h_{2}\right|^{2}+\sigma^{2}\right)+\sigma^{2}\right\}+k_{1}}$.

Further simplification of (58) leads to

$$
\gamma_{1}^{T W F D}=\frac{e_{1} x y}{x\left[f_{1}\left(P_{1} x+P_{2} y+g_{1}\right)+g_{1}\right]+k_{1}} .
$$

This finishes the proof of (21). The same procedure is applied to prove (22).

\section{Appendix C}

This section will prove the formulas in (26) and (27). First of all, we start with

$$
\begin{aligned}
P_{\text {out }, 1}^{\text {OWFD }} & =\operatorname{Pr}\left(\gamma_{1}^{\text {OWFD }}<\tau\right) \\
& =\operatorname{Pr}\left(\frac{a_{2} x y}{b x+c_{2} x y+c}<\tau\right) \\
& =\operatorname{Pr}\left(a_{2} x y<\tau\left\{b x+c_{2} x y+c\right\}\right) \\
& =\operatorname{Pr}\left(a_{2} x y-\tau c_{2} x y<\tau\{b x+c\}\right) \\
& =\left\{\begin{array}{cc}
\operatorname{Pr}\left(y<\frac{\tau\{b x+c\}}{x\left(a_{2}-\tau c_{2}\right)}\right) & , a_{2}-\tau c_{2}>0 \\
1 & , a_{2}-\tau c_{2}<0
\end{array}\right.
\end{aligned}
$$

We further simplify (60) for the case of $a_{2}-\tau c_{2}>0$ as

$$
\begin{aligned}
P_{\text {out }, 1}^{O W F D} & =\int_{0}^{\infty} F_{Y}\left(\frac{\tau\{b x+c\}}{x\left(a_{2}-\tau c_{2}\right)}\right) f_{X}(x) d x \\
& =1-\int_{0}^{\infty} e^{-\frac{\tau\{b x+c\}}{x\left(a_{2}-\tau c_{2}\right)} \lambda_{2}} \lambda_{1} e^{-\lambda_{1} x} d x \\
& =1-\lambda_{1} e^{-\frac{\lambda_{2} \tau b}{\left(a_{2}-\tau c_{2}\right)}} \int_{0}^{\infty} e^{-\frac{4 \tau c \lambda_{2}}{\left(a_{2}-\tau c_{2}\right) 4 x}-\lambda_{1} x} d x \\
& =1-\lambda_{1} e^{-\frac{\lambda_{2} \tau b}{\left(a_{2}-\tau c_{2}\right)}} \int_{0}^{\infty} e^{-\frac{\psi}{4 x}-\lambda_{1} x} d x \\
& =1-\lambda_{1} e^{-\frac{\lambda_{2} \tau b}{\left(a_{2}-\tau c_{2}\right)}} \sqrt{\frac{\psi}{\lambda_{1}}} K_{1}\left(\sqrt{\psi \lambda_{1}}\right) .
\end{aligned}
$$

This finished the proof of (26). The same procedure is applied to prove (27).

In the following, we will prove the formulas in (37) and (38). We start with

$$
\begin{aligned}
& P_{\text {out }, 1}^{\text {TWFD }}=\operatorname{Pr}\left(\gamma_{1}^{T W F D}<\tau\right) \\
& =\operatorname{Pr}\left(\frac{e_{1} x y}{x\left[f_{1}\left(P_{1} x+P_{2} y+g_{1}\right)+g_{1}\right]+k_{1}}<\tau\right) \\
& =\operatorname{Pr}\left(e_{1} x y<\tau x\left[f_{1}\left(P_{1} x+P_{2} y+g_{1}\right)+g_{1}\right]+\tau k_{1}\right) \\
& =\operatorname{Pr}\left(e_{1} x y<\tau f_{1} P_{2} x y+\tau f_{1} x\left\{P_{1} x+g_{1}\right\}+\tau x g_{1}+\tau k_{1}\right) \\
& =\operatorname{Pr}\left(x y\left\{e_{1}-\tau f_{1} P_{2}\right\}\right)<\tau x\left(f_{1} P_{1} x+f_{1} g_{1}+g_{1}\right)+\tau k_{1}
\end{aligned}
$$




$$
\begin{aligned}
\gamma_{1}^{T W F D}= & \frac{P_{2}\left|h_{1}\right|^{2}\left|h_{2}\right|^{2}}{P_{R}\left|h_{1}\right|^{2}\left|h_{r r}\right|^{2}+\left|h_{1}\right|^{2} \sigma_{R}^{2}+\frac{P_{1}\left|h_{11}\right|^{2}+\sigma_{1}^{2}}{\theta^{2} P_{R}}} \\
= & \frac{P_{2}\left|h_{1}\right|^{2}\left|h_{2}\right|^{2}}{P_{R}\left|h_{1}\right|^{2}\left|h_{r r}\right|^{2}+\left|h_{1}\right|^{2} \sigma_{R}^{2}+\frac{\left(P_{1}\left|h_{11}\right|^{2}+\sigma_{1}^{2}\right)\left(P_{1}\left|h_{1}\right|^{2}+P_{2}\left|h_{2}\right|^{2}+P_{R}\left|h_{r r}\right|^{2}+\sigma_{R}^{2}\right)}{P_{R}}} \\
= & \frac{P_{2}\left|h_{1}\right|^{2}\left|h_{2}\right|^{2}}{P_{R}\left|h_{1}\right|^{2}\left|h_{r r}\right|^{2}+\left|h_{1}\right|^{2} \sigma_{R}^{2}+\frac{\left(P_{1}\left|h_{11}\right|^{2}+\sigma_{1}^{2}\right)\left(\frac{P_{R}}{\phi}+P_{R}\left|h_{r r}\right|^{2}\right)}{P_{R}}} \\
= & \frac{P_{2}\left|h_{1}\right|^{2}\left|h_{2}\right|^{2}}{P_{R}\left|h_{1}\right|^{2}\left|h_{r r}\right|^{2}+\left|h_{1}\right|^{2} \sigma_{R}^{2}+\left(P_{1}\left|h_{11}\right|^{2}+\sigma_{1}^{2}\right)\left(\frac{1}{\phi}+\left|h_{r r}\right|^{2}\right)}
\end{aligned}
$$

and

$$
\begin{aligned}
& P_{\text {out }, 1}^{\text {TWFD }}= \\
& =\left\{\begin{array}{cc}
\operatorname{Pr}\left(y<\frac{\tau x\left(f_{1} P_{1} x+f_{1} g_{1}+g_{1}\right)+\tau k_{1}}{x\left(e_{1}-\tau f_{1} P_{2}\right)}\right) & , e_{1}-\tau f_{1} P_{2}>0 \\
1 & , e_{1}-\tau f_{1} P_{2}<0
\end{array}\right.
\end{aligned}
$$

Now, we compute $P_{\text {out, }}^{T W F D}$ in (63) for the case of $e_{1}-\tau f_{1} P_{2}>0$ as

$$
\begin{aligned}
& P_{\text {out }, 1}^{T W F D}= \\
& =\int_{0}^{\infty} F_{Y}\left(\frac{\tau x\left(f_{1} P_{1} x+f_{1} g_{1}+g_{1}\right)+\tau k_{1}}{x\left(e_{1}-\tau f_{1} P_{2}\right)}\right) f_{X}(x) d x \\
& =1-\int_{0}^{\infty} e^{-\lambda_{2} \frac{\tau\left(f_{1} P_{1} x+f_{1} g_{1}+g_{1}\right)}{e_{1}-\tau f_{1} P_{2}}-\lambda_{2} \frac{\tau k_{1}}{x\left(e_{1}-\tau f_{1} P_{2}\right)} \lambda_{1} e^{-\lambda_{1} x} d x} \\
& =1-\lambda_{1} e^{-\frac{\lambda_{2} \tau\left(f_{1} g_{1}+g_{1}\right)}{e_{1}-\tau f_{1} P_{2}}} \int_{0}^{\infty} e^{-\frac{\lambda_{2} \tau k_{1}}{\left(e_{1}-\tau f_{1} P_{2}\right) x}-\frac{\lambda_{2} \tau f_{1} P_{1}}{e_{1}-\tau f_{1} P_{2}} x-\lambda_{1} x} d x \\
& =1-\lambda_{1} e^{-\frac{\lambda_{2} \tau\left(f_{1} g_{1}+g_{1}\right)}{e_{1}-\tau f_{1} P_{2}}} \int_{0}^{\infty} e^{-\frac{4 \lambda_{2} \tau k_{1}}{\left(e_{1}-\tau f_{1} P_{2}\right) 4 x}-\left(\frac{\lambda_{2} \tau f_{1} P_{1}}{e_{1}-\tau f_{1} P_{2}}+\lambda_{1}\right) x} d x \\
& =1-\lambda_{1} e^{-\frac{\lambda_{2} \tau\left(f_{1} g_{1}+g_{1}\right)}{e_{1}-\tau f_{1} P_{2}}} \int_{0}^{\infty} e^{-\frac{\Omega_{1}}{4 x}-\Psi_{1} x} d x \\
& \left.=1-\lambda_{1} e^{-\frac{\lambda_{2} \tau\left(f_{1} g_{1}+g_{1}\right)}{e_{1}-\tau f_{1} P_{2}}} \sqrt{\frac{\Omega_{1}}{\Psi_{1}} K_{1}\left(\sqrt{\Omega_{1}} \Psi_{1}\right.}\right) .
\end{aligned}
$$

This finished the proof of (37). The same procedure is applied to prove (38).

\section{Appendix D}

For the AF based TWHD communications, the SNRs were given by [38] and [39] as $\gamma_{1}=\frac{a X Y}{b X+c}$ and $\gamma_{2}=$ $\frac{d X Y}{b Y+c}$. Then, the OP of the TWHD communications is

$$
\begin{aligned}
P_{\text {out }}^{T W H D}= & \operatorname{Pr}\left(\left\{\gamma_{1}<\tau\right\} \cup\left\{\gamma_{2}<\tau\right\}\right) \\
= & \underbrace{\operatorname{Pr}\left(\gamma_{1}<\tau\right)}_{A_{1}}+\underbrace{\operatorname{Pr}\left(\gamma_{2}<\tau\right)}_{A_{2}} \\
& -\underbrace{\operatorname{Pr}\left(\left\{\gamma_{1}<\tau\right\} \cap\left\{\gamma_{2}<\tau\right\}\right)}_{A_{3}}
\end{aligned}
$$

where

$$
\begin{aligned}
& A_{1}=\operatorname{Pr}\left(\gamma_{1}<\tau\right) \\
& =\operatorname{Pr}\left(\frac{a X Y}{b X+c}<\tau\right) \\
& =\operatorname{Pr}(a X Y<\tau\{b X+c\}) \\
& =\operatorname{Pr}\left(Y<\frac{\tau\{b X+c\}}{a X}\right) \\
& =\int_{0}^{\infty} F_{Y}\left(\frac{\tau\{b X+c\}}{a X}\right) f_{X}(x) d x \\
& =\int_{0}^{\infty}\left(1-e^{-\lambda_{2} \frac{\tau\{b X+c\}}{a X}}\right) \lambda_{1} e^{-\lambda_{1} x} d y \\
& =1-\int_{0}^{\infty} e^{-\lambda_{2} \frac{\tau\{b X+c\}}{a X}} \lambda_{1} e^{-\lambda_{1} x} d x \\
& =1-\lambda_{1} e^{-\frac{\lambda_{2} b \tau}{a}} \int_{0}^{\infty} e^{-\frac{\lambda_{2} c \tau}{a x}-\lambda_{1} x} d x \\
& =1-\lambda_{1} e^{-\frac{\lambda_{2} b \tau}{a}} \int_{0}^{\infty} e^{-\frac{4 \lambda_{2} c \tau}{a 4 x}-\lambda_{1} x} d x \\
& =1-\lambda_{1} e^{-\frac{\lambda_{2} b \tau}{a}} \sqrt{\frac{4 \lambda_{2} c \tau}{a \lambda_{1}}} K_{1}\left(\sqrt{\frac{4 \lambda_{1} \lambda_{2} c \tau}{a}}\right)
\end{aligned}
$$

and

$$
\begin{aligned}
A_{2} & =\operatorname{Pr}\left(\gamma_{2}<\tau\right) \\
& =1-\lambda_{2} e^{-\frac{\lambda_{1} b \tau}{d}} \sqrt{\frac{4 \lambda_{1} c \tau}{d \lambda_{2}}} K_{1}\left(\sqrt{\frac{4 \lambda_{1} \lambda_{2} c \tau}{d}}\right) .
\end{aligned}
$$

The derivation of $A_{3}$ below can use to the proof of 
the OP formula in (29) for $P_{\text {out }, 12}^{O W F D}$ in the OWFD scheme and (41) for $P_{\text {out,12 }}^{T W F D}$ in the TWFD scheme as

$$
\begin{aligned}
A_{3} & =\operatorname{Pr}\left(\left\{\gamma_{1}<\tau\right\} \cap\left\{\gamma_{2}<\tau\right\}\right) \\
& =\operatorname{Pr}\left(\left\{\frac{a x y}{b x+c}<\tau\right\} \cap\left\{\frac{d x y}{b y+c}<\tau\right\}\right) \\
& =\operatorname{Pr}\left(\left\{y<\frac{\tau(b x+c)}{a x}\right\} \cap\left\{x<\frac{\tau(b y+c)}{d y}\right\}\right) \\
& =\int_{0}^{x_{0}} \int_{\frac{y_{0}}{x_{0}} x}^{\frac{\tau(b x+c)}{a x}} f_{x, y}(x, y) d y d x+\int_{0}^{y_{0}} \int_{\frac{x_{0}}{y_{0}} y}^{\frac{\tau(b y+c)}{d y}} f_{x, y}(x, y) d x d y \\
& =\mathcal{P}_{1}+\mathcal{P}_{2},
\end{aligned}
$$

where $x_{0}=\frac{\varphi+\sqrt{\varphi^{2}+4 \tau a b^{2} c}}{2 a b}, y_{0}=\frac{\tau\left(b x_{0}+c\right)}{a x_{0}}$, and $\varphi=$ $-a c+\tau b^{2}+c d$.

The formula in (68) can be rewritten as

$$
\begin{aligned}
& \mathcal{P}_{1}=\int_{0}^{x_{0}} \int_{\frac{y_{0}}{x_{0}} x}^{\frac{\tau(b x+c)}{a x}} f_{x, y}(x, y) d y d x \\
& =\int_{0}^{x_{0}} \int_{\frac{y_{0}}{x_{0}} x}^{\frac{\tau(b x+c)}{a x}} \lambda_{1} e^{-\lambda_{1} x} \lambda_{2} e^{-\lambda_{2} y} d y d x \\
& =\lambda_{1} \lambda_{2} \int_{0}^{x_{0}}\left[\int_{\frac{y_{0}}{x_{0}} x}^{\frac{\tau(b x+c)}{a x}} e^{-\lambda_{2} y} d y\right] e^{-\lambda_{1} x} d x \\
& =-\lambda_{1} \int_{0}^{x_{0}}\left(e^{-\frac{\lambda_{2} \tau(b x+c)}{a x}}-e^{-\frac{\lambda_{2} y_{0}}{x_{0}} x}\right) e^{-\lambda_{1} x} d x \\
& =-\lambda_{1} \int_{0}^{x_{0}} e^{-\frac{\lambda_{2} \tau(b x+c)}{a x}} e^{-\lambda_{1} x} d x+\lambda_{1} \int_{0}^{x_{0}} e^{-\frac{\lambda_{2} y_{0}}{x_{0}} x} e^{-\lambda_{1} x} d x \\
& =-\lambda_{1} e^{-\frac{\lambda_{2} b \tau}{a}} \int_{0}^{x_{0}} e^{-\frac{\lambda_{2} \tau c}{a x}} e^{-\lambda_{1} x} d x+\lambda_{1} \int_{0}^{x_{0}} e^{-\frac{\lambda_{2} y_{0}}{x_{0}} x} e^{-\lambda_{1} x} d x \\
& =\underbrace{-\lambda_{1} e^{-\frac{\lambda_{2} b \tau}{a}} \int_{0}^{x_{0}} e^{-\frac{\lambda_{2} \tau c}{a x}} e^{-\lambda_{1} x} d x}_{\mathcal{T}_{1}}+\underbrace{\lambda_{1} \int_{0}^{x_{0}} e^{-\frac{\lambda_{2} y_{0}}{x_{0}} x} e^{-\lambda_{1} x} d x}_{\mathcal{T}_{2}}
\end{aligned}
$$

The $\mathcal{T}_{2}$ term is given by

$$
\begin{aligned}
\mathcal{T}_{2} & =\lambda_{1} \int_{0}^{x_{0}} e^{-\frac{\lambda_{2} y_{0}}{x_{0}} x} e^{-\lambda_{1} x} d x \\
& =\lambda_{1} \int_{0}^{x_{0}} e^{-\left(\frac{\lambda_{2} y_{0}}{x_{0}}+\lambda_{1}\right) x} d x \\
& =-\frac{\lambda_{1}}{\frac{\lambda_{2} y_{0}}{x_{0}}+\lambda_{1}}\left(e^{-\left(\frac{\lambda_{2} y_{0}}{x_{0}}+\lambda_{1}\right) x_{0}}-1\right)
\end{aligned}
$$

and

$$
\begin{aligned}
\mathcal{T}_{1} & =-\lambda_{1} e^{-\frac{\lambda_{2} b \tau}{a}} \int_{0}^{x_{0}} e^{-\frac{\lambda_{2} \tau c}{a x}} e^{-\lambda_{1} x} d x \\
& =-\lambda_{1} e^{-\frac{\lambda_{2} b \tau}{a}}\left(\int_{0}^{\infty} e^{-\frac{\lambda_{2} \tau c}{a x}} e^{-\lambda_{1} x} d x-\int_{x_{0}}^{\infty} e^{-\frac{\lambda_{2} \tau c}{a x}} e^{-\lambda_{1} x} d x\right) \\
& =-\lambda_{1} e^{-\frac{\lambda_{2} b \tau}{a}}(\underbrace{\int_{0}^{\infty} e^{-\frac{\lambda_{2} \tau c}{a x}-\lambda_{1} x} d x}_{\mathcal{T}_{11}}-\underbrace{}_{\mathcal{T}_{12} e^{\int_{0}} e^{-\frac{\lambda_{2} \tau c}{a x}} e^{-\lambda_{1} x} d x}) .
\end{aligned}
$$

Applying [40, Eq. (3.324.1)], we obtain

$$
\begin{aligned}
\mathcal{T}_{11} & =\int_{0}^{\infty} e^{-\frac{\lambda_{2} \tau c}{a x}-\lambda_{1} x} d x \\
& =\int_{0}^{\infty} e^{-\frac{4 \lambda_{2} \tau c}{a 4 x}-\lambda_{1} x} d x \\
& =\int_{0}^{\infty} e^{-\frac{\beta_{1}}{4 x}-\lambda_{1} x} d x \\
& =\sqrt{\frac{\beta_{1}}{\lambda_{1}}} K_{1}\left(\sqrt{\beta_{1} \lambda_{1}}\right),
\end{aligned}
$$

where $\beta_{1}=\frac{4 \lambda_{2} \tau c}{a}$.

We rewrite $\mathcal{T}_{12}$ in $(71)$ as

$$
\begin{aligned}
\mathcal{T}_{12} & =\int_{x_{0}}^{\infty} e^{-\frac{\lambda_{2} \tau c}{a x}} e^{-\lambda_{1} x} d x \\
& =\int_{x_{0}}^{\infty} e^{-\frac{\phi_{1}}{x}} e^{-\lambda_{1} x} d x .
\end{aligned}
$$

Let $\phi_{1}=\frac{\lambda_{2} \tau c}{a}$. Then, applying the Taylor series expansion for $e^{-\frac{\phi_{1}}{x}}=\sum_{t=0}^{\infty} \frac{(-1)^{t} \phi_{1}{ }^{t}}{t ! x^{t}}$, one obtain

$$
\begin{aligned}
\mathcal{T}_{12} & =\int_{x_{0}}^{\infty} \sum_{t=0}^{\infty} \frac{(-1)^{t} \phi_{1}^{t}}{t ! x^{t}} e^{-\lambda_{1} x} d x \\
& =\sum_{t=0}^{\infty} \frac{(-1)^{t} \phi_{1}{ }^{t}}{t !} \int_{x_{0}}^{\infty} \frac{e^{-\lambda_{1} x}}{x^{t}} d x .
\end{aligned}
$$

Using the exponent integral

$$
E_{k}(z)=\int_{1}^{\infty} \frac{e^{-z t}}{t^{k}} d t
$$

to write (74) in the closed form as

$$
\mathcal{T}_{12}=\sum_{t=0}^{\infty} \frac{(-1)^{t} \phi_{1}^{t}}{t !}\left(x_{0}\right)^{1-k} E_{k}\left(\lambda_{1} x_{0}\right) \text {. }
$$


From (69), we have

$$
\begin{aligned}
\mathcal{P}_{1}= & \mathcal{T}_{1}+\mathcal{T}_{2} \\
= & -\lambda_{1} e^{-\frac{\lambda_{2} b \tau}{a}}\left(\mathcal{T}_{11}+\mathcal{T}_{12}\right) \\
& -\frac{\lambda_{1}}{\lambda_{2} y_{0} / x_{0}+\lambda_{1}}\left(e^{-\left(\lambda_{2} y_{0}+\lambda_{1} x_{0}\right)}-1\right) \\
= & -\lambda_{1} e^{-\frac{\lambda_{2} b \tau}{a}}\left(\sqrt{\frac{\beta_{1}}{\lambda_{1}}} K_{1}\left(\sqrt{\beta_{1} \lambda_{1}}\right)\right. \\
& \left.-\sum_{t=0}^{\infty} \frac{(-1)^{t} \phi_{1}^{t}}{t !}\left(x_{0}\right)^{1-t} E_{t}\left(\lambda_{1} x_{0}\right)\right) \\
& -\frac{\lambda_{1}}{\lambda_{2} y_{0} / x_{0}+\lambda_{1}}\left(e^{-\left(\lambda_{2} y_{0}+\lambda_{1} x_{0}\right)}-1\right) .
\end{aligned}
$$

Following the same derivation as $\mathcal{P}_{1}$, we have skipped some manipulation of $\mathcal{P}_{2}$ in (78)

$$
\begin{aligned}
\mathcal{P}_{2} & =\int_{0}^{y_{0}} \int_{\frac{x_{0}}{y_{0}} y}^{\frac{\tau(b y+c)}{d y}} f_{x, y}(x, y) d x d y \\
& =\int_{0}^{y_{0}} \int_{\frac{\tau(b y+c)}{d y}}^{x_{0} y} \lambda_{1} e^{-\lambda_{1} x} \lambda_{2} e^{-\lambda_{2} y} d x d y \\
& =\underbrace{-\lambda_{2} e^{-\frac{\lambda_{1} b \tau}{d}} \int_{0}^{y_{0}} e^{-\frac{\lambda_{1} \tau c}{d y}} e^{-\lambda_{2} y} d y}_{H_{1}}+\underbrace{\lambda_{0}^{y_{0} e_{0}^{-\frac{\lambda_{1} x_{0}}{y_{0}} y} e^{-\lambda_{2} y} d y}}_{H_{2}}
\end{aligned}
$$

where

$$
\begin{aligned}
H_{2} & =\lambda_{2} \int_{0}^{y_{0}} e^{-\frac{\lambda_{1} x_{0}}{y_{0}} y} e^{-\lambda_{2} y} d y \\
& =-\frac{\lambda_{2}}{\lambda_{1} x_{0} / y_{0}+\lambda_{2}}\left(e^{-\left(\lambda_{1} x_{0}+\lambda_{2} y_{0}\right)}-1\right)
\end{aligned}
$$

and

$$
\begin{aligned}
H_{1} & =-\lambda_{2} e^{-\frac{\lambda_{1} b \tau}{d}} \int_{0}^{y_{0}} e^{-\frac{\lambda_{1} \tau c}{d y}} e^{-\lambda_{2} y} d y \\
& =-\lambda_{2} e^{-\frac{\lambda_{1} b \tau}{d}}(\underbrace{\int_{0}^{\infty} e^{-\frac{\lambda_{1} \tau c}{d y}-\lambda_{2} y} d y}_{H_{11}}+\underbrace{\int_{x y}^{\infty} e^{-\frac{\lambda_{1} \tau c}{d y}} e^{-\lambda_{2} y} d y}_{H_{12}})
\end{aligned}
$$

with

$$
\begin{aligned}
H_{11} & =\int_{0}^{\infty} e^{-\frac{\lambda_{1} \tau c}{d y}-\lambda_{2} y} d y \\
& =\sqrt{\frac{\beta_{2}}{\lambda_{2}}} K_{1}\left(\sqrt{\beta_{2} \lambda_{2}}\right),
\end{aligned}
$$

where $\beta_{2}=\frac{4 \lambda_{1} \tau c}{d}$ and

$$
\begin{aligned}
H_{12} & =\int_{y_{0}}^{\infty} e^{-\frac{\lambda_{1} \tau c}{d y}} e^{-\lambda_{2} y} d y \\
& =\int_{y_{0}}^{\infty} e^{-\frac{\phi_{2}}{y}} e^{-\lambda_{2} y} d y .
\end{aligned}
$$

Let $\phi_{2}=\frac{\lambda_{1} \tau c}{d}$. Then, we have $e^{-\frac{\phi_{2}}{y}}=\sum_{t=0}^{\infty} \frac{(-1)^{t} \phi_{2}^{t}}{t ! y^{t}}$ and

$$
\begin{aligned}
H_{12} & =\int_{y_{0}}^{\infty} \sum_{t=0}^{\infty} \frac{(-1)^{t} \phi_{2}{ }^{t}}{t ! y^{t}} e^{-\lambda_{2} y} d y \\
& =\sum_{t=0}^{\infty} \frac{(-1)^{t} \phi_{2}{ }^{t}}{t !} \int_{y_{0}}^{\infty} \frac{e^{-\lambda_{2} y}}{y^{t}} d y \\
& =\sum_{t=0}^{\infty} \frac{(-1)^{t} \phi_{2}{ }^{t}}{t !}\left(y_{0}\right)^{1-t} E_{t}\left(\lambda_{2} y_{0}\right)
\end{aligned}
$$

Inserting (79) and (80) into (78), we have

$$
\begin{aligned}
\mathcal{P}_{2}= & H_{1}+H_{2} \\
= & -\lambda_{2} e^{-\frac{\lambda_{1} b \tau}{d}}\left(\sqrt{\frac{\beta_{2}}{\lambda_{2}}} K_{1}\left(\sqrt{\beta_{2} \lambda_{2}}\right)-\right. \\
& \left.\sum_{t=0}^{\infty} \frac{(-1)^{t} \phi_{2}{ }^{t}}{t !}\left(y_{0}\right)^{1-t} E_{t}\left(\lambda_{2} y_{0}\right)\right) \\
& -\frac{\lambda_{2}}{\lambda_{1} x_{0} / y_{0}+\lambda_{2}}\left(e^{-\left(\lambda_{1} x_{0}+\lambda_{2} y_{0}\right)}-1\right) .
\end{aligned}
$$

Therefore, combining (77) with (84) results in

$$
\begin{aligned}
A_{3}= & \operatorname{Pr}\left(\left\{\gamma_{1}<\tau\right\} \cap\left\{\gamma_{2}<\tau\right\}\right) \\
= & -\lambda_{1} e^{-\frac{\lambda_{2} b \tau}{a}}\left(\sqrt{\frac{\beta_{1}}{\lambda_{1}}} K_{1}\left(\sqrt{\beta_{1} \lambda_{1}}\right)\right. \\
& \left.-\sum_{t=0}^{\infty} \frac{(-1)^{t} \phi_{1}^{t}}{t !}\left(x_{0}\right)^{1-t} E_{t}\left(\lambda_{1} x_{0}\right)\right) \\
& -\frac{\lambda_{1}}{\lambda_{2} y_{0} / x_{0}+\lambda_{1}}\left(e^{-\left(\lambda_{2} y_{0} / x_{0}+\lambda_{1}\right)}-1\right) \\
& -\lambda_{2} e^{-\frac{\lambda_{1} b \tau}{d}}\left(\sqrt{\frac{\beta_{2}}{\lambda_{2}}} K_{1}\left(\sqrt{\beta_{2} \lambda_{2}}\right)\right. \\
& \left.-\sum_{t=0}^{\infty} \frac{(-1)^{t} \phi_{2}{ }^{t}}{t !}\left(y_{0}\right)^{1-t} E_{t}\left(\lambda_{2} y_{0}\right)\right) \\
& -\frac{\lambda_{2}}{\lambda_{1} x_{0} / y_{0}+\lambda_{2}}\left(e^{-\left(\lambda_{1} x_{0} / y_{0}+\lambda_{2}\right)}-1\right) .
\end{aligned}
$$

This finishes the proof of $P_{\text {out }, 12}^{O W F D}$ in (29) and $P_{\text {out }, 12}^{T W F D}$ in (41).

\section{REFERENCES}

[1] H. Van Toan, V.-N. Q. Bao, and H. Nguyen-Le, “Cognitive two-way relay systems with multiple primary receivers: exact and asymptotic outage formulation," IET Communications, vol. 11, no. 16, pp. 2490-2497, 2017.

[2] H. Van Toan, V. N. Q. Bao, and K. N. Le, "Performance 
analysis of cognitive underlay two-way relay networks with interference and imperfect channel state information," EURASIP Journal on Wireless Communications and Networking, vol. 53 (2018), no. 1, 2018.

[3] V. N. Q. Bao, H. Van Toan, and K. N. Le, "Performance of two-way AF relaying with energy harvesting over Nakagami-m fading channels," IET Communications, vol. 12, no. 20, pp. 2592-2599, 2018.

[4] P. Nguyen-Huu, H.-V. Khuong, and V.-N. Q. Bao, "Secrecy outage analysis of energy harvesting two-way relaying networks with friendly jammer," IET Communications, vol. 13, no. 13, pp. 1877-1885, 2019.

[5] A. H. Gazestani, S. A. Ghorashi, B. Mousavinasab, and M. Shikh-Bahaei, "A survey on implementation and applications of full duplex wireless communications," Physical Communication, vol. 34, pp. 121-134, 2019.

[6] X. Sun, D. Zhang, and X. Dai, "Performance analysis of full-duplex based two-way relaying," China Communications, vol. 13, no. 11, pp. 35-48, 2016.

[7] G. Liu, F. R. Yu, H. Ji, V. C. Leung, and X. Li, "Inband full-duplex relaying: A survey, research issues and challenges," IEEE Communications Surveys $\mathcal{E}$ Tutorials, vol. 17, no. 2, pp. 500-524, 2015.

[8] Z. Zhang, Z. Ma, M. Xiao, G. K. Karagiannidis, Z. Ding, and P. Fan, "Two-timeslot two-way full-duplex relaying for 5G wireless communication networks," IEEE Transactions on Communications, vol. 64, no. 7, pp. 2873-2887, 2016.

[9] Z. Zhang, Z. Ma, Z. Ding, M. Xiao, and G. K. Karagiannidis, "Full-duplex two-way and one-way relaying: average rate, outage probability, and tradeoffs," IEEE Transactions on Wireless Communications, vol. 15, no. 6, pp. 3920-3933, 2016.

[10] A. Rauniyar, P. Engelstad, and O. N. Østerbø, "Performance analysis of $\mathrm{rf}$ energy harvesting and information transmission based on noma with interfering signal for iot relay systems," IEEE Sensors Journal, 2019.

[11] Q. Zeng, Y. Zheng, B. Zhong, and Z. Zhang, "Minimum transmission protocol for full-duplex systems with energy harvesting," IEEE Communications Letters, vol. 23, no. 2, pp. 382-385, 2018.

[12] D. Zhai, R. Zhang, J. Du, Z. Ding, and F. R. Yu, "Simultaneous wireless information and power transfer at 5G new frequencies: Channel measurement and network design," IEEE Journal on Selected Areas in Communications, vol. 37, no. 1, pp. 171-186, 2018.

[13] X. Ge, H. Jia, Y. Zhong, Y. Xiao, Y. Li, and B. Vucetic, "Energy Efficient Optimization of Wireless-powered 5G Full Duplex Cellular Networks: A Mean Field Game Approach," IEEE Transactions on Green Communications and Networking, vol. 3, no. 2, pp. 455-467, 2019.

[14] Y. Wang, K. Xu, A. Liu, and X. Xia, "Hybrid oneway full-duplex/two-way half-duplex relaying scheme," IEEE Access, vol. 5, pp. 7737-7745, 2017.

[15] X. Song and S. Xu, "Joint optimal power allocation and relay selection in full-duplex energy harvesting relay networks," in Proceedings of the 10th International Conference on Communication Software and Networks (ICCSN). IEEE, 2018, pp. 80-84.

[16] Q. N. Le, V. N. Q. Bao, and B. An, "Full-duplex distributed switch-and-stay energy harvesting selection relaying networks with imperfect CSI: Design and outage analysis," Journal of Communications and Networks, vol. 20, no. 1, pp. 29-46, 2018.

[17] G. Nauryzbayev, M. Abdallah, and K. M. Rabie, "Outage probability of the EH-Based Full-Duplex AF and DF Relaying Systems in $\alpha-\mu$ environment," in Proceedings of the 88th Vehicular Technology Conference (VTC-Fall). IEEE, 2018, pp. 1-6.

[18] X. Xie, J. Chen, and Y. Fu, “Outage performance and qos optimization in full-duplex system with non-linear energy harvesting model," IEEE Access, vol. 6, pp. $44281-$ 44290, 2018.
[19] R. Chen and H. Zhang, "Power efficiency optimisation of wireless-powered full-duplex relay systems," IET Communications, vol. 12, no. 5, pp. 603-611, 2018.

[20] J. Gong, X. Chen, and M. Xia, "Transmission optimization for hybrid half/full-duplex relay with energy harvesting," IEEE Transactions on Wireless Communications, vol. 17, no. 5, pp. 3046-3058, 2018.

[21] D. Wang, R. Zhang, X. Cheng, and L. Yang, “Full-duplex energy-harvesting relay networks: Capacity-maximizing relay selection," Journal of Communications and Information Networks, vol. 3, no. 3, pp. 79-85, 2018.

[22] Ö. T. Demir and T. E. Tuncer, "Robust optimum and near-optimum beamformers for decode-and-forward full-duplex multi-antenna relay with self-energy recycling," IEEE Transactions on Wireless Communications, vol. 18, no. 3, pp. 1566-1580, 2019.

[23] A. A. Nasir, H. D. Tuan, T. Q. Duong, and H. V. Poor, "Full-duplex MIMO-OFDM communication with selfenergy recycling," arXiv preprint arXiv:1903.09931, 2019.

[24] T. Nam Thanh, D. Dinh-Thuan, and M. Voznak, "Fullduplex cognitive radio NOMA networks: Outage and throughput performance analysis," International Journal of Electronics and Telecommunications, vol. 65, no. 1, pp. 103-109, 2019.

[25] Y. Wang, B. Xia, and Z. Chen, "Performance analysis of two-way full-duplex amplify-forward relay systems," in Proceedings of the 26th Annual International Symposium on Personal, Indoor, and Mobile Radio Communications (PIMRC). IEEE, 2015, pp. 492-496.

[26] J. H. Moon, J. J. Park, and D. I. Kim, “Energy signal design and decoding procedure for full-duplex two-way wireless powered relay," in Proceedings of the URSI AsiaPacific Radio Science Conference (URSI AP-RASC). IEEE, 2016, pp. 442-445.

[27] D. Wang, R. Zhang, X. Cheng, and L. Yang, "Relay selection in two-way full-duplex energy-harvesting relay networks," in Proceedings of the IEEE Global Communications Conference (GLOBECOM). IEEE, 2016, pp. 1-6.

[28] A. Koc, I. Altunbas, and E. Basar, "Two-way full-duplex spatial modulation systems with wireless powered af relaying," IEEE Wireless Communications Letters, vol. 7, no. 3, pp. 444-447, 2017.

[29] X.-X. Nguyen and D.-T. Do, "Bidirectional communication in full duplex wireless-powered relaying networks: Time-switching protocol and performance analysis," Wireless Personal Communications, vol. 98, no. 1, pp. 879-896, 2018.

[30] C. Li, H. Wang, Y. Yao, Z. Chen, X. Li, and S. Zhang, "Outage performance of the full-duplex two-way DF relay system under imperfect CSI," IEEE Access, vol. 5, pp. 5425-5435, 2017.

[31] X. Song, Y. Ni, X. Han, and S. Xu, "Optimal power splitting of full duplex wireless powered communication networks with two-way relay," in Proceedings of the 3rd International Conference on Mechanical, Control and Computer Engineering (ICMCCE). IEEE, 2018, pp. 374-378.

[32] W. Wang, L. An, R. Wang, L. Yin, and G. Zhang, "Maxmin fair beamforming designs of swipt-aided full-duplex two-way relay systems," Physical Communication, vol. 29, pp. 22-30, 2018.

[33] G. Chen, P. Xiao, J. R. Kelly, B. Li, and R. Tafazolli, "Fullduplex wireless-powered relay in two way cooperative networks," IEEE Access, vol. 5, pp. 1548-1558, 2017.

[34] T. Riihonen, S. Werner, and R. Wichman, "Hybrid fullduplex/half-duplex relaying with transmit power adaptation," IEEE Transactions on Wireless Communications, vol. 10, no. 9, pp. 3074-3085, 2011.

[35] D. Wang, R. Zhang, X. Cheng, and L. Yang, "Full-duplex energy-harvesting relay networks: Capacity-maximizing relay selection," Journal of Communications and Information Networks, vol. 3, no. 3, pp. 79-85, 2018.

[36] H. Cui, M. Ma, L. Song, and B. Jiao, "Relay selection for 
two-way full duplex relay networks with amplify-andforward protocol," IEEE Transactions on Wireless Communications, vol. 13, no. 7, pp. 3768-3777, 2014.

[37] H. Chen, G. Li, and J. Cai, "Spectral-energy efficiency tradeoff in full-duplex two-way relay networks," IEEE Systems Journal, vol. 12, no. 1, pp. 583-592, 2015.

[38] Z. Chen, B. Xia, and H. Liu, "Wireless information and power transfer in two-way amplify-and-forward relaying channels," in Proceedings of the Global Conference on Signal and Information Processing (GlobalSIP). IEEE, 2014, pp. 168-172.

[39] Y. Liu, L. Wang, M. Elkashlan, T. Q. Duong, and A. Nallanathan, "Two-way relay networks with wireless power transfer: design and performance analysis," IET Communications, vol. 10, no. 14, pp. 1810-1819, 2016.

[40] A. Jeffrey and D. Zwillinger, Table of integrals, series, and products. Elsevier, 2007.

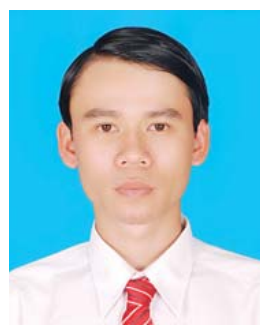

Phong Nguyen-Huu received the B.E. degree in Telecommunications Engineering from University of Transport and Communi cationsCampus II (UTC2), Vietnam in 2006, and the Master's degree in Telecommunications Engineering from Posts and Tele communications Institute of Technology (PTIT), Vietnam in 2014. Currently, he is working towards a Ph.D. degree in Ho Chi Minh City University of Technology (HCMUT), Vietnam. His research interests are two-way communications, fullduplex transmission, and energy harvesting.

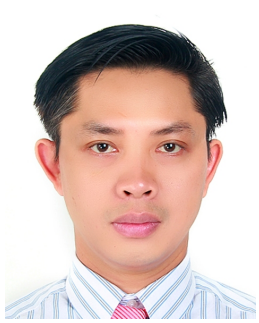

Khuong Ho-Van received the B.E. (with the first-rank honor) and the M.S. degrees in Electronics and Telecommunications Engineering from Ho Chi Minh City University of Technology, Vietnam, in 2001 and 2003, respectively, and the Ph.D. degree in Electrical Engineering from University of Ulsan, Korea in 2007. During 2007-2011, he joined McGill University, Canada as a postdoctoral fellow. Currently, he is an Associate professor at Ho Chi Minh City University of Technology. His major research interests are modulation and coding techniques, diversity techniques, digital signal processing, energy harvesting, physical layer security, and cognitive radio.

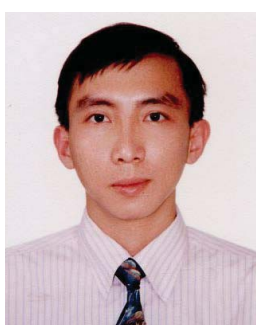

Vo Nguyen Quoc Bao was born in Nha Trang, Khanh Hoa Province, Vietnam. He received the B.E. and M.Eng. degree in electrical engineering from Ho Chi Minh City University of Technology (HCMUT), Vietnam, in 2002 and 2005, respectively, and Ph.D. degree in electrical engineering from University of Ulsan, South Korea, in 2009. In 2002, he joined the Department of Electrical Engineering, Posts and Telecommunications Institute of Technology (PTIT), as a lecturer. Since February 2010,

he has been with the Department of Telecommunications, PTIT, where he is currently an Assistant Professor. His major research interests are modulation and coding techniques, MIMO systems, combining techniques, cooperative communications, and cognitive radio. Dr. Bao is a member of Korea Information and Communications Society (KICS), The Institute of Electronics, Information and Communication Engineers (IEICE) and The Institute of Electrical and Electronics Engineers (IEEE). He is currently serving as the Editor of Transactions on Emerging Telecommunications Technologies (Wiley ETT). He is also a Guest Editor of EURASIP Journal on Wireless Communications and Networking, special issue on "Cooperative Cognitive Networks" and IET Communications, special issue on "Secure Physical Layer Communications". 